\title{
LOCAL ANALYSIS OF HYBRID SYSTEMS ON POLYHEDRAL SETS WITH STATE-DEPENDENT SWITCHING
}

\author{
JOHN LETH, RAFAEL WISNIEWSKI
}

Department of Electronic Systems, Automation and Control Aalborg University, Fredrik Bajers Vej 7 C, 9220 Aalborg East, Denmark e-mail: \{jjl, raf\}@es.aau.dk

\begin{abstract}
This paper deals with stability analysis of hybrid systems. Various stability concepts related to hybrid systems are introduced. The paper advocates a local analysis. It involves the equivalence relation generated by reset maps of a hybrid system. To establish a tangible method for stability analysis, we introduce the notion of a chart, which locally reduces the complexity of the hybrid system. In a chart, a hybrid system is particularly simple and can be analyzed with the use of methods borrowed from the theory of differential inclusions. Thus, the main contribution of this paper is to show how stability of a hybrid system can be reduced to a specialization of the well established stability theory of differential inclusions. A number of examples illustrate the concepts introduced in the paper.
\end{abstract}

Keywords: stability, switched systems, hybrid systems, differential inclusions.

\section{Introduction}

In this article, we study the notion of stability for a class of hybrid systems with state-dependent switching. In short, a hybrid system consists of a family of dynamical systems with discrete transitions realized by reset maps. To this end, we bring simple examples illustrating various dynamical phenomena which characterize this class of hybrid systems. In particular, we show that, due to the underlying topology and reset maps, one has to consider non-equivalent notions of stability in order to grasp the asymptotic behavior of the system. We provide examples that illustrate two non-equivalent stable behavior: the local version where stability is confined to a single polyhedral set due to the "directions" of reset maps, and the global version where "directions" of reset maps do not play any role. The latter corresponds to the standard definition of stability (Lygeros et al., 2003; Goebel and Teel, 2006).

Before providing a detailed description of the content of this paper, we survey the research field related to the findings.

Hybrid systems comprise a rich family of dynamical systems (Liberzon, 2003; van der Schaft and Schumacher, 2000; Tabuada, 2009; Haddad et al., 2006), e.g., non-smooth dynamics (Leine and Nijmeijer, 2004; Kunze, 2000), arising from friction, backlash or collision, can be seen as a hybrid system. Moreover, many real world systems are subjected to discrete transitions. In particular, van der Schaft and Schumacher (2000) give a number of illustrative examples from various application areas, such as transmission control and variable structure systems, Tomlin et al. (1998) use hybrid system formalism to control air traffic, Wisniewski and Leth (2011) model a system with hysteresis as a piecewise-affine hybrid system, Ding et al. (2011) discuss the use of the hybrid system formalism in robotics, whereas Balluchi et al. (2005) identify challenges and opportunities for hybrid systems in automotive industry. Furthermore, estimation of hybrid systems is addressed by Rienmüller et al. (2013).

Stability of hybrid systems has been studied before (Lygeros et al., 2003; Goebel and Teel, 2006; Goebel et al., 2009), and classical concepts of stability and asymptotic stability were adopted to hybrid systems. Indeed, this generalization was possible because a single state space of the hybrid system was allowed, whereas in this article, as in the works of Simić et al. (2005), Ames and Sastry (2005) or Bujorianu and Lygeros (2006), a family of state spaces is permitted. Of the above references, that by Simic et al. (2005) is mostly related to this work, which also constructs a single state space in the process of gluing along reset maps. Nonetheless, contrary to Simić et al. (2005), we allow equilibria on the boundary 
of the state spaces. As a consequence, the concepts of stability become more subtle.

Even though the time-dependent switching paradigm is not considered in this work, which concentrates on state-depending switching, we mention the stability analysis of state-varying switched systems under time-dependent switching (Yang et al., 2013), since it can be seen as a parallel study in the time-dependent case.

Stability of hybrid systems with state-dependent switching has also been studied before (Leth and Wisniewski, 2012; Pettersson and Lennartson, 2002; Johansson and Rantzer, 1998). The class of hybrid systems considered in the literature was restricted to be defined on a single state space-a subset of Euclidean space partitioned by polyhedral sets. In this paper, such hybrid systems are called switched systems (with state-dependent switching) following the terminology by Liberzon (2003, Chapter 3.3). Asymptotic stability of switched systems was addressed by Leth and Wisniewski (2012), who applied the theory of differential inclusions to formulate a mathematical framework for studying their dynamic behavior. In the works of Johansson and Rantzer (1998) as well as Pettersson and Lennartson (2002), algorithms for finding piecewise quadratic Lyapunov functions for asymptotically stable switched systems were devised. In both approaches, the sought Lyapunov functions were solutions to certain linear matrix inequalities.

Before proceeding to the description of the contents of this work, we add a remark on applicability of the chosen class of hybrid systems. The restriction to polyhedral sets, as described in the above paragraph, arises "naturally" in engineering applications, for example, in the presence of saturations (Rantzer and Johansson, 2000), for control synthesis of piecewise affine systems (Yordanov et al., 2012), and hysteresis-control (Wisniewski and Leth, 2011). Moreover, the usage of this system (under the name of piecewise-linear systems) as a systematic approach to numerical nonlinear control was advocated by Sontag (1981) (see also Heemels et al., 2001). A control method for such systems (this time, under the name of piecewise-affine hybrid systems) was developed by Habets and van Schuppen (2005). The advantage of choosing this approach is reflected in simplicity of implementation, theoretical analysis, and calculation. Moreover, Yang et al. (2011) used polyhedral partitioning of the state space to facilitate control synthesis with the aim of stabilizing a switched system under time-dependent switching.

We proceed with the description of the contents of this work. We analyze a hybrid system consisting of a family of dynamical systems defined on disjoint polyhedral sets and discrete transitions, realized by reset maps defined on the facets of these polyhedral sets. Likewise, a trajectory of a hybrid system is a disjoint union of flow lines of the constituent dynamical systems. The start and end points of the flow lines on the polyhedral sets are related by the reset maps. A hybrid system as described above can be locally analyzed in the interior of each of the polyhedral sets employing standard methods from the theory of dynamical systems.

For instance, if an equilibrium is contained in the interior of a polyhedral set, then Lyapunov stability theory and its descendants apply. The situation becomes immediately more complex if an equilibrium point lies on a facet. To this end, we follow Simić et al. (2005), Ames and Sastry (2005) as well as Wisniewski (2006), and regard the reset maps as generators of an equivalence relation which allows gluing the polyhedral sets together. The result of this construction is a quotient system. We show that stability of the original hybrid system and that of the quotient system are related: the hybrid system is asymptotically (quotient) stable if and only if its quotient system is.

The behavior of a hybrid system can be overly complicated; however, by imposing certain regularity conditions, the methods from dynamical systems can be applied. This is the reason for introducing the concept of a chart. A chart is a map from a set in the state space of a hybrid system (with a non-empty interior) onto a single polyhedral set. In a chart, a hybrid system is particularly simple - it is a switched system. It consists of a single state space, which is partitioned by polyhedral sets. The dynamical model is given by a differential inclusion, which reduces to a single valued map in the interior of each cell of the partition.

Using the concept of a chart, we give necessary and sufficient conditions for asymptotic stability of an equilibrium point of a particularly regular hybrid system. This implies that the stability analysis can be carried out locally; hence, standard methods can be applied.

The paper is organized as follows. Section 2 introduces terminology and preliminaries used throughout this paper. A definition and stability results for switched systems are recalled in Section 3. In Section 4, a hybrid system and its trajectories are formally defined. Furthermore, motivated by a number of examples, various concepts of stability are developed. The notion of a chart is defined and its properties are devised in Section 5 It is shown that, in a chart, a hybrid system is merely a switched system (with state-dependent switching). Moreover, regular and omnidirectional hybrid systems are introduced, and it is shown that asymptotic stability in a chart is equivalent to (quotient) stability of the original regular omnidirectional hybrid system.

\section{Preliminaries}

In this section, we bring in the notation and terminology used in the article. For concepts and results related to 
polyhedral sets and polytopes, we refer the reader to Appendix.

2.1. Notation and terminology. Let $k \in \mathbb{N} \cup\{\infty\}$; if $k \in \mathbb{N}$, let $\mathbf{k}$ denote the set $\{1,2, \ldots, k\}$, otherwise $\mathbf{k}=\mathbb{N}$. Moreover, by $\mathbf{k}_{0}$ we denote the set $\mathbf{k} \cup\{0\}$, and by $\mathbf{k}^{*}$ the set $\mathbf{k}-\{k\}$ if $k \in \mathbb{N}$, otherwise $\mathbf{k}^{*}=\mathbf{k}$.

Occasionally, we will consider sets, say $\left\{P_{i}\right\}$, where some of the elements are "copies of each other" meaning that they are distinguished by their index alone, e.g., $P_{k}=$ $S \times\{k\}$ and $P_{j}=S \times\{j\}$ for some set $S$. By abuse of notation, we indicate this by writing $P_{i}=S, i=k, j$.

Given a subset $U$ of a topological space $X$, by $\operatorname{cl}_{X}(U), \operatorname{int}_{X}(U)$ and $b_{X}(U)$, we denote respectively the closure, the interior and the boundary of $U$ in $X$. The notation $\operatorname{cl}(U), \operatorname{int}(U)$ and $\operatorname{bd}(U)$ is also used if the space $X$ is clear from the context.

The disjoint union of a family of topological spaces $\left\{X_{i}\right\}$ is the space $\bigsqcup_{i} X_{i}=\bigcup_{i}\left(X_{i} \times\{i\}\right)$ with the topology making each $X_{i} \times\{i\}$ open and closed, and each inclusion $X_{i} \times\{i\} \hookrightarrow \bigsqcup_{i} X_{i}$ a homeomorphism to its image. By abuse of notation, we sometimes write $X_{i}$ in place of the subspace $X_{i} \times\{i\} \subset \bigsqcup_{i} X_{i}$. Moreover, an element $(x, i) \in \bigsqcup_{i} X_{i}$ will sometimes be written as $(x ; i)$ or simply $x$.

2.1.1. Polyhedral complex. Let $K=\left\{P_{j} \mid j \in I\right\}$ be a collection of polyhedral sets for some index set $I$. We define $|K|=\bigcup_{i \in I} P_{i} \subset \mathbb{R}^{n}$, with the subspace topology.

We say that $K$ is a (polyhedral) complex if (i) $P \in$ $K$ implies that any $F \prec P$ is also an element of $K$, (ii) $P, Q \in K$ implies that $P \cap Q \prec P$ and $P \cap Q \prec Q$, and (iii) any point of $|K|$ has a neighborhood intersecting only finitely many elements of $K$. If $I$ is finite, as assumed in the following, then the last condition is automatically satisfied. Let $E$ be any polyhedral set ( $\mathbb{R}^{n}$ inclusive). A piecewise linear partition (or, for short, partitioning) of $E$ is a complex $K$ such that $E=|K|$. Note that any polyhedral set $P$ induces, in a canonical way, a (convex) complex $\{F \mid F \preceq P\}$. This and other standard facts from the theory of convex polytopes (Grünbaum, 2003) will be used throughout without further mentioning.

Let $K$ be a complex with index set $I$. We define $I^{j}=$ $\left\{i \in I \mid \operatorname{dim}\left(P_{i}\right)=j\right\}$ and $K^{j}=\left\{P_{i} \in K \mid i \in I^{j}\right\}$.

\section{Switched systems with state-dependent switching}

In this section, we discuss a special class of hybrid systems called switched systems with state-dependent switching. Such systems were studied by Leth and Wisniewski (2012) under the name of switched systems. The purpose of introducing them will become clear in Section 5, where it is shown that some hybrid systems can be modeled locally as a switched system with state-dependent switching.

Definition 1. (Switched system with state-dependent switching) A switched system with state-dependent switching (of dimension $n$ ) is a triple $(Q, K, \mathcal{F})$, where

- $Q$ is a polyhedral set in $\mathbb{R}^{n}$,

- $K=\left\{P_{j} \mid j \in I\right\}$ is a (piecewise linear) partition of $Q$ with finite index set $I$,

- $\mathcal{F}=\left\{f_{i}: P_{i} \rightarrow \mathbb{R}^{n} \mid i \in I^{n}\right\}$ is a family of smooth functions.

We use the word "smooth" in the above definition in the following sense: $f_{i}$ is the restriction of a smooth map $\mathbb{R}^{n} \rightarrow \mathbb{R}^{n}$. In the sequel, we usually write "switched system" in place of the more cumbersome "switched system state-dependent switching".

Analysis of a switched system is cast in the framework of differential inclusions. The dynamics of the switched system $(Q, K, \mathcal{F})$ are governed by the following differential inclusions:

$$
\dot{x}(t) \in F^{c}(x(t)),
$$

where $\dot{x}=\mathrm{d} x / \mathrm{d} t$ and $F^{c}$ is the convexification of the set valued map $F$ defined by

$$
F: Q \rightarrow 2^{\mathbb{R}^{n}} ; x \mapsto\left\{v \in \mathbb{R}^{n} \mid v=f_{i}(x) \text { if } x \in P_{i}\right\},
$$

with $2^{\mathbb{R}^{n}}$ being the power set of $\mathbb{R}^{n}$.

Now, let $(Q, K, \mathcal{F})$ denote a switched system of dimension $n$. From the theory of ordinary differential equations, we conclude that at any interior point $x$ of $Q$ there exists a solution at $x$, i.e., there exist $0<$ $T<\infty$ and an absolutely continuous function $[0, T] \rightarrow$ $E^{\prime} ; t \mapsto x(t)$, which solves the Cauchy problem $\dot{x}(t) \in$ $F^{c}(x(t))$ a.e. for $x(0)=x$.

Moreover, if $x$ is interior to a $P \in K^{n}$, then the solution is a classical one, i.e., there exist $0<T<$ $\infty$ and a continuously differentiable function $[0, T] \rightarrow$ $E^{\prime} ; t \mapsto x(t)$, which solves the Cauchy problem $\dot{x}(t) \in$ $F^{c}(x(t)), x(0)=x$.

After Leth and Wisniewski (2012, Proposition 1 and Theorem 1(b)), we conclude the following.

Proposition 1. Let $(Q, K, \mathcal{F})$ denote a switched system of dimension $n$, and $T_{Q}(x)$ the contingent cone to $Q$ at $x$, i.e., the closure of the convex cone of $Q-\{x\}$. For each unbounded $P_{i} \in K^{n}$, assume that $f_{i}\left(P_{i}\right), f_{i} \in \mathcal{F}$, is bounded. Then at any $x \in Q$ there exists a solution defined on $[0, \infty)$

(i) if $Q=\mathbb{R}^{n}$,

(ii) iff $F^{c}(x) \cap T_{Q}(x) \neq \emptyset$ for all $x \in Q$, in the case $Q \neq \mathbb{R}^{n}$. 
Recall that an equilibrium $x_{*}$, i.e., $0 \in F^{c}\left(x_{*}\right)$, is $F^{c}$-stable (resp. weakly $F^{c}$-stable), if for each $\epsilon>0$ there exists $\delta>0$ such that

$$
\left|x-x_{*}\right|<\delta \Rightarrow\left|x(t)-x_{*}\right|<\epsilon, \quad \forall t \in[0, \infty),
$$

for each (resp. some) solution at $x$. An equilibrium point which is not weakly $F^{c}$-stable is called $F^{c}$-unstable. An equilibrium point $x_{*}$ is called asymptotically $F^{c}$-stable (resp. weakly asymptotically $F^{c}$-stable) if it is $F^{c}$-stable (resp. weakly $F^{c}$-stable) and $x(t) \rightarrow x_{*}$ for $t \rightarrow \infty$. We remark that $F^{c}$-stable is called stable by Leth and Wisniewski (2012). This change in nomenclature is to avoid confusion with the notion of stability presented in Section 4.1.1

Proposition 2. Assume that $0 \in F^{c}(0)$. If there exists $r>0$ and continuous positive definite and negative definite functions $v: \mathbb{R}^{n} \rightarrow \mathbb{R}$ and $w: \mathbb{R}^{n} \rightarrow \mathbb{R}$, respectively, such that for each $x$, with $|x|<r$,

$$
D^{+} v(x)(u)=\limsup _{\substack{h \rightarrow 0^{+} \\ u^{\prime} \rightarrow u}} \frac{v(x+h u)-v(x)}{h} \leq w(x),
$$

for all $u \in F^{c}(x)$, then the equilibrium point 0 is asymptotically $F^{c}$-stable. Moreover, the equilibrium point 0 is $F^{c}$-stable if $w$ is negative semi-definite.

The following example, borrowed from Leth and Wisniewski (2012), illustrates that a solution can converge to an equilibrium point in finite time (which cannot happen for solutions of ordinary differential equations with a Lipschitz continuous right-hand side). Moreover, it will serve as a motivation for concepts introduced later in Section 4

Example 1. Consider the switched system $(Q, K, \mathcal{F})$ where $Q=\mathbb{R}^{2}, K^{2}=\left\{P_{i}\right\}_{i \in I^{2}}$ with $I^{2}=\{1,2,3,4\}$ and $P_{i}$ being the $i$-th quadrant. Let $\mathcal{F}=\left\{f_{i}\right\}$ with $f_{1}$, $f_{2}, f_{3}$ and $f_{4}$ being the constant vector fields $(-2,1)$, $(-1,-1),(1,-1)$ and $(1,1)$, respectively.

It follows that the unique solution $t \mapsto x(t)$ to (1) at $x=(2,0)$ is defined on $[0,8]$, that it "spirals" towards the origin $\left(\lim _{t \rightarrow 8} x(t)=0\right)$, and that it switches infinitely many times from $f_{i}$ to $f_{i+1}$ (here $f_{4+1}=f_{1}$ ). That is at each time instant $t_{j+1}=$ $4 \sum_{i=0}^{j} 1 / 2^{i}, j \in\{0,1,2, \ldots\}$, with the corresponding state instant $x\left(t_{j+1}\right)=\left(1 / 2^{j}, 0\right)$, the system switches from $f_{4}$ to $f_{1}$, and $t_{j+1} \rightarrow 8, x\left(t_{j+1}\right) \rightarrow(0,0)$ as $j \rightarrow \infty$.

Since each solution can be extended trivially to $[0, \infty)$ at the origin, it follows that $x_{*}=0$ is an asymptotically $F^{c}$-stable equilibrium point. Note, however that before the convexification (replace $F^{c}$ by $F$ in (10) $x_{*}$ was not even an equilibrium point; in fact, no solution exists at $x_{*}=0$.

\section{Hybrid systems}

We introduce a class of hybrid systems defined on a family of polyhedral sets. Each such system consists of a family of dynamical systems equipped with a collection of reset maps realizing discrete transition between these dynamical systems. Formally, we consider the class of hybrid systems expressed as a triple $(\mathcal{P}, \mathcal{S}, \mathcal{R})$, where

- $\mathcal{P}=\mathcal{P}_{J}$ is a finite family of polyhedral sets:

$$
\begin{array}{r}
\mathcal{P}=\left\{P_{j} \subset \mathbb{R}^{n} \mid P_{j}\right. \text { being a polyhedral set, } \\
\left.\operatorname{dim}\left(P_{j}\right)=n, j \in J\right\},
\end{array}
$$

with $J$ as a finite index set;

- $\mathcal{S}$ is a (finite) family of smooth vector fields:

$$
\mathcal{S}=\left\{\xi_{j}: P_{j} \rightarrow \mathbb{R}^{n} \mid P_{j} \in \mathcal{P}, j \in J\right\} ;
$$

- $\mathcal{R}=\mathcal{R}_{L}$ is a finite family of polyhedral maps, called reset maps:

$$
\begin{gathered}
\mathcal{R}=\left\{R_{l}: F \rightarrow F^{\prime} \mid F \prec P \in \mathcal{P}, F^{\prime} \prec P^{\prime} \in \mathcal{P},\right. \\
\left.\operatorname{dim}(F)=\operatorname{dim}\left(F^{\prime}\right)=n-1, l \in L\right\},
\end{gathered}
$$

with $L$ being a finite index set.

In what follows, we refer to elements of this class simply as hybrid systems instead of the more precise but awkward name: hybrid systems on polyhedral sets with state-dependent switching. Note that a such a system can be regarded as an automaton with discrete modes corresponding to affine systems living on polyhedral sets.

The formalism of a hybrid system allows for the existence of facets where no transition occurs. Furthermore, a hybrid system $(\mathcal{P}, \mathcal{S}, \mathcal{R})$ has in a natural way associated a di-graph $(\mathcal{P}, \mathcal{E})$, where an edge $e=$ $\left(P, P^{\prime}\right) \in \mathcal{E} \subset \mathcal{P} \times \mathcal{P}$ exists whenever there is a reset map $R: F \rightarrow F^{\prime}$ in $\mathcal{R}$ such that $F \prec P$ and $F^{\prime} \prec P^{\prime}$. As a consequence, the definition of a hybrid system presented here is a special case of that presented by Goebel $e t$ al. (2009) and Haddad et al. (2006), where each vertex of a transition system has assigned a dynamical system.

In the rest of this paper, it is assumed that for each $j \in J$ and each $x \in P_{j}$ the solution to the Cauchy problem $x^{\prime}(t)=\xi(x(t)), x(0)=x$ does not exhibit blow up behavior, i.e., $0<T<\infty$ does not exists such that $t \mapsto x(t)$ is defined on $[0, T)$, and $\lim _{t \rightarrow T}|x(t)|=\infty$. Note that this assumption is irrelevant if $P_{j}$ is a polytope.

For a hybrid system $\left(\mathcal{P}_{J}, \mathcal{S}, \mathcal{R}\right)$, let $\sim \subset X \times X$ with $X=\bigsqcup_{j \in J} P_{j}$ be the smallest equivalence relation containing the relations $p \sim^{\prime} R(p)$ for points $p \in \operatorname{dom}(R)$ and a reset map $R \in \mathcal{R}$. The equivalence class containing $N \subset X$ will be denoted by $[N]$, and $\pi: X \rightarrow X^{*}$, with $X^{*}=X / \sim$, denotes the canonical (quotient) map. 
We now turn to some basic properties of reset maps and show how these are related to the equivalence relation $\sim$. To this end, we fix a hybrid system $(\mathcal{P}, \mathcal{S}, \mathcal{R})$ with $\mathcal{P}$ consisting of polytopes only. Let $\mathcal{R}^{0}=\mathcal{R} \cup \mathcal{R}^{-1}$ with $\mathcal{R}^{-1}=\left\{R^{-1} \mid R \in \mathcal{R}\right\}$. By Lemma A1, in Appendix, $\mathcal{R}^{0}$ consists of polyhedral maps. If $R_{1}, R_{2} \in \mathcal{R}^{0}$ and $V=\operatorname{im}\left(R_{2}\right) \cap \operatorname{dom}\left(R_{1}\right) \neq \emptyset$, then we define $R_{1} \diamond R_{2}$ : $R_{2}^{-1}(V) \rightarrow R_{1}(V)$ by $R_{1} \diamond R_{2}(x)=R_{1}\left(R_{2}(x)\right)$. Since the reset maps are polyhedral, $\operatorname{dom}\left(R_{1} \diamond R_{2}\right)$ and $\operatorname{im}\left(R_{1} \diamond\right.$ $R_{2}$ ) are faces of some polyhedral sets in $\mathcal{P}$. We proceed inductively and define a set $\mathcal{R}^{1}$ of finite compositions of maps belonging to $\mathcal{R}^{0}$ :

$$
R \in \mathcal{R}^{1} \Leftrightarrow R=R_{1} \diamond \ldots \diamond R_{k}, \quad k \in \mathbb{N},
$$

where $R_{i} \in \mathcal{R}^{0}$ for $i \in \mathbf{k}$, and $\operatorname{im}\left(R_{i+1}\right) \cap \operatorname{dom}\left(R_{i}\right) \neq \emptyset$ for $i \in \mathbf{k}^{*}$. Hence the map $R$ in (2) is injective. Moreover, it follows that the relation between the elements of $\mathcal{R}^{1}$ and the equivalence relation $\sim$ is $x \sim y$ if and only if there is $R \in \mathcal{R}^{1}$ such that $R(x)=y$.

In the next lemma, we prove that it is also polyhedral if $\mathcal{P}$ consists of polytopes.

Lemma 1. Let $(\mathcal{P}, \mathcal{S}, \mathcal{R})$ be a hybrid system with $\mathcal{P}$ consisting of polytopes only. Any map $R \in \mathcal{R}^{1}$ is polyhedral. Furthermore, $\operatorname{dom}(R)$ and $\operatorname{im}(R)$ are faces of some polytope in $\mathcal{P}$.

Proof. We prove Lemma 1 by induction on the length $k$ in (2). By definition, the reset maps are polyhedral, and by Lemma A1 so are their inverse maps. Furthermore, by the same lemma, the domain and the image of a reset map are facets of some polytopes in $\mathcal{P}$. Thus, the lemma is true for $k=1$.

For the inductive step, let $R=R_{1} \diamond \ldots \diamond R_{k} \diamond R_{k+1}$, where $\operatorname{dom}(R) \subset P \in \mathcal{P}$ and $\operatorname{im}(R) \subset P^{\prime \prime} \in \mathcal{P}$. By the induction hypothesis, $\bar{R}=R_{1} \diamond \ldots \diamond R_{k}$ is a polyhedral map, and $\operatorname{dom}(\bar{R})$ and $\operatorname{im}(\bar{R})$ are faces of $P^{\prime}$ and $P^{\prime \prime}$ in $\mathcal{P}$, respectively. Let $V=\operatorname{im}\left(R_{k+1}\right) \cap \operatorname{dom}(\bar{R}) \neq \emptyset$. Hence, $V \preceq \operatorname{im}\left(R_{k+1}\right)$ and $V \preceq \operatorname{dom}(\bar{R})$ since $P^{\prime}$ is a complex. We show that the map $\bar{R} \diamond R_{k+1}: R_{k+1}^{-1}(V) \rightarrow \hat{R}(V)$ is polyhedral.

By Lemma A1, the map $R_{k+1}^{-1}$ is polyhedral; hence, $R_{k+1}^{-1}(V) \preceq \operatorname{dom}\left(R_{k+1}\right)$. Let $F \preceq R_{k+1}^{-1}(V)$ then $F \preceq$ $\operatorname{dom}\left(R_{k+1}\right)$. Therefore, by the inductive assumption, $R_{k+1}(F) \preceq \operatorname{im}\left(R_{k+1}\right)$. Since $R_{k+1}(F) \subset V$, we have $R_{k+1}(F) \preceq V$. Now $R(F)=\bar{R}\left(R_{k+1}(F)\right)$ is a face of $\operatorname{im}(\bar{R})$, by the induction hypothesis; thus, it is also a face of $\bar{R}(V)$ and $P^{\prime \prime}$. The dimensions of $F$ and $R(F)$ are the same since $R$ is an injection.

Corollary 1. $\mathcal{R}^{1}$ consists of a finite number of distinct maps.

Proof. The corollary follows from the following two observations: The sets $\mathcal{P}$ and $\mathcal{R}$ are finite, and the family of faces of a polyhedral set is finite.
4.1. Trajectories of a hybrid system. We need the following technicality in order to introduce the concept of a hybrid system trajectory. Let $k \in \mathbb{N} \cup\{\infty\}$ be fixed. A subset $\mathcal{T}_{k} \subset \mathbb{R}_{+} \times \mathbb{Z}_{+}$will be called a $k$-time domain (or simply a time domain) if there exists a non-decreasing sequence $\left\{t_{i}\right\}_{i \in \mathbf{k}_{0}}$ in $\mathbb{R}_{+} \cup\{\infty\}$ such that $\mathcal{T}_{k}=\bigsqcup_{i \in \mathbf{k}} T_{i}$, where $T_{i}=\left[t_{i-1}, t_{i}\right]$ if either $i \in \mathbf{k}^{*}$ or $i=k$ and $t_{k}<$ $\infty$, otherwise $T_{k}=\left[t_{k-1}, \infty\right)=\left[t_{k-1}, t_{k}\right)$.

In particular, $T_{i}=\left[t_{i-1}, t_{i}\right]$ for all $i \in \mathbf{k}$ whenever $k=\infty$; see Example [3] The sequence $\left\{t_{i}\right\}_{i \in \mathbf{k}_{0}}$ corresponding to a $k$-time domain will be called a switching sequence, a name justified by Definition 2

Now, we introduce the concept of a trajectory of a hybrid system. It is the concatenation of trajectories induced by vector fields of the hybrid system by means of its reset maps. A similar definition was put forward by Goebel and Teel (2006).

Definition 2. (k-trajectory) A $k$-trajectory, or just a trajectory, of the hybrid system $\left(\mathcal{P}_{J}, \mathcal{S}, \mathcal{R}_{L}\right)$ is a pair $\left(\mathcal{T}_{k}, \gamma\right)$, where $k \in \mathbb{N} \cup\{\infty\}$ is fixed, and

- $\mathcal{T}_{k} \subset \mathbb{R}_{+} \times \mathbb{Z}_{+}$is a time domain with the corresponding switching sequence $\left\{t_{i}\right\}_{i \in \mathbf{k}_{0}}$, such that $\operatorname{int}_{\mathbb{R}}\left(T_{i}\right) \neq \emptyset$ for at least one $T_{i} \in \mathcal{T}_{k}$;

- $\gamma: \mathcal{T}_{k} \rightarrow \bigsqcup_{j \in J} P_{j}$ is continuous and satisfies:

(i) For each $i \in \mathbf{k}^{*}$, there exists a pair $\left\{j, j^{\prime}\right\} \subset J$ with $j \neq j^{\prime}$ and such that $\gamma\left(t_{i} ; i\right) \in \operatorname{bd}\left(P_{j}\right)$, and $\gamma\left(t_{i} ; i+1\right) \in \operatorname{bd}\left(P_{j^{\prime}}\right)$;

(ii) for each $i \in \mathbf{k}$, there exists $j \in J$ such that $\gamma(t ; i) \in P_{j}$ for all $t \in T_{i}$, and $\frac{\partial}{\partial t} \gamma(t ; i)=$ $\xi_{j}(\gamma(t ; i))$ for almost all $t \in T_{i}$;

(iii) for each $i \in \mathbf{k}^{*}$, there exists $l \in L$ such that $R_{l}\left(\gamma\left(t_{i} ; i\right)\right)=\gamma\left(t_{i} ; i+1\right)$.

A trajectory at $(x ; j), j \in J$, is a trajectory $\left(\mathcal{T}_{k}, \gamma\right)$ with $\gamma\left(t_{0} ; 1\right)=(x ; j)$.

In the above definition, property (ii) is vacuous whenever $t_{i-1}=t_{i}$. Furthermore, $k-1$ is the number of discrete transitions that a $k$-trajectory makes. To avoid purely discrete behavior, that is, trajectories with only discrete transitions, we have imposed the premise $\operatorname{int}_{\mathbb{R}}\left(T_{i}\right) \neq \emptyset$ for at least one $T_{i}$. Note that the exclusion of this premise does not change the results of this paper, and it would make the formulations and proofs awkward with separate treatment in this case; reckon for instance, Proposition 9 Nonetheless, if a precise analysis of the space of trajectories is to be conducted, it is important to include such trajectories due to the completeness property: the limits of trajectories have to be trajectories. This subject is not the matter of study in this paper.

Unless explicitly mentioned otherwise, we let, in the sequel, $t_{0}=0$ and write $\dot{\gamma}$ instead of $\frac{\partial \gamma}{\partial t}$, and $\gamma(t)$ instead of $\gamma(t ; j)$ whenever the index $j$ is irrelevant. Moreover, 
we will always assume that trajectories are maximal, that is, if $\left(\mathcal{T}_{k}, \gamma\right)$ is a trajectory, then there exists no trajectory $\left(\mathcal{T}_{k^{\prime}}^{\prime}, \gamma^{\prime}\right)$ such that $k \leq k^{\prime}, \mathcal{T}_{k} \subsetneq \mathcal{T}_{k^{\prime}}^{\prime}$ and $\gamma\left(\mathcal{T}_{k}\right)=\gamma^{\prime}\left(\mathcal{T}_{k}\right)$.

4.1.1. Local and global stability. Now, we define the notion of stability for hybrid systems. A point $x_{*} \in$ $X=\bigsqcup_{j \in J} P_{j}$ is called an equilibrium point of the hybrid system $\left(\mathcal{P}_{J}, \mathcal{S}, \mathcal{R}_{L}\right)$, if $\xi\left(x_{*}\right)=0$ for some $\xi \in \mathcal{S}$ (recall our notation convention, $\left.x_{*}=\left(x_{*} ; i\right)\right)$.

An equilibrium $x_{*}$ is said to be locally stable (resp. locally weak stable) if for each open neighborhood $V \subset$ $X$ of $x_{*}$ there exists an open neighborhood $U \subset V$ of $x_{*}$ such that for each $x \in U$ and for each (resp. some) trajectory $\left(\mathcal{T}_{k}, \gamma\right)$ at $x$ we have $\gamma(t) \in V$ for all $t \in \mathcal{T}_{k}$. An equilibrium point which is not locally weak stable is called locally unstable.

An equilibrium point $x_{*}$ is called locally asymptotically stable (resp. locally weak asymptotically stable) if it is locally stable (resp. locally weak stable) and $\gamma(t) \rightarrow x_{*}$ for $t \rightarrow \infty$.

Example 2. Let $(\mathcal{P}, \mathcal{S}, \mathcal{R})$ be a hybrid system with $\mathcal{P}=$ $\left\{P_{1}, P_{2}\right\}$, where $P_{i}=\mathbb{R}_{+} \times \mathbb{R}, i=1,2$, and $\mathcal{S}=$ $\left\{\xi_{1}, \xi_{2}\right\}$, where $\xi_{1}(x)=\left(-x_{1},-x_{2}\right)$ and $\xi_{2}(x)=(1,0)$. Suppose that there is one reset map id $: \operatorname{bd}_{\mathbb{R}^{2}}\left(P_{1}\right) \rightarrow$ $\operatorname{bd}_{\mathbb{R}^{2}}\left(P_{2}\right)$, the identity.

The origin $x_{*}=0=(0 ; 1)$ (in $P_{1}$ ) is clearly an equilibrium point, and for each open neighborhood $V \subset$ $X$ of $x_{*}$ there exists an open neighborhood $U \subset V$ of $x_{*}$ (take $\left.U=V \cap P_{1}\right)$ such that for some trajectory $\left(\mathcal{T}_{k}, \gamma\right.$ ) at $x \in U$ we have $\gamma(t) \in V$ for all $t \in \mathcal{T}_{k}=[0, \infty)$, and $\gamma(t) \rightarrow x_{*}$ for $t \rightarrow \infty$. Hence, the origin is locally weak asymptotically stable; however, it is not locally asymptotically stable since at each $x \in \operatorname{bd}_{\mathbb{R}^{2}}\left(P_{1}\right)$ there exists a trajectory which immediately jumps to $P_{2}$ and stays there.

Note that the origin becomes locally asymptotically stable if the reset map is replaced by id : $\operatorname{bd}_{\mathbb{R}^{2}}\left(P_{2}\right) \rightarrow$ $\operatorname{bd}_{\mathbb{R}^{2}}\left(P_{1}\right)$.

Due to the topology on $X$ and the reset maps, local stability is not always adequate for describing the behavior of a hybrid system, as the next example illustrates.

Example 3. Let $J=\{1,2,3,4\}$, and let $\left(\mathcal{P}_{J}, \mathcal{S}, \mathcal{R}_{J}\right)$ be a hybrid system with $\mathcal{P}=\left\{P_{i}\right\}_{i \in J}$, where $P_{i}$ is the $i$-th quadrant in $\mathbb{R}^{2}, \mathcal{S}=\left\{\xi_{i}\right\}$, where $\xi_{i}$ is the restriction of the asymptotically stable system $x \mapsto\left(-x_{1}-x_{2}, x_{1}-x_{2}\right)$ to $P_{i}$, and $\mathcal{R}=\left\{R_{i}\right\}$, where

$$
\begin{aligned}
& \left\{x \in \mathrm{bd}_{\mathbb{R}^{2}}\left(P_{1}\right) \mid x_{1}=0\right\} \stackrel{R_{1}=\mathrm{id}}{\longrightarrow}\left\{x \in \mathrm{bd}_{\mathbb{R}^{2}}\left(P_{2}\right) \mid x_{1}=0\right\}, \\
& \left\{x \in \mathrm{bd}_{\mathbb{R}^{2}}\left(P_{2}\right) \mid x_{2}=0\right\} \stackrel{R_{2}=\mathrm{id}}{\longrightarrow}\left\{x \in \mathrm{bd}_{\mathbb{R}^{2}}\left(P_{3}\right) \mid x_{2}=0\right\}, \\
& \left\{x \in \mathrm{bd}_{\mathbb{R}^{2}}\left(P_{3}\right) \mid x_{1}=0\right\} \stackrel{R_{3}=\mathrm{id}}{\longrightarrow}\left\{x \in \mathrm{bd}_{\mathbb{R}^{2}}\left(P_{4}\right) \mid x_{1}=0\right\}, \\
& \left\{x \in \mathrm{bd}_{\mathbb{R}^{2}}\left(P_{4}\right) \mid x_{2}=0\right\} \stackrel{R_{4}=\mathrm{id}}{\longrightarrow}\left\{x \in \mathrm{bd}_{\mathbb{R}^{2}}\left(P_{1}\right) \mid x_{2}=0\right\} .
\end{aligned}
$$

Then the equilibrium $x_{*}=0=(0 ; i), i \in J$, is locally unstable, since we may take as $V$ the intersection of $P_{i}$ with any open neighborhood of 0 in $\mathbb{R}^{2}$. However, globally the dynamical behavior of the hybrid system exhibits a kind of asymptotically stable behavior, in that if $U$ is any open neighborhood of $x_{*}$ and $\left(\mathcal{T}_{k}, \gamma\right)$ is a trajectory then for any $t^{\prime} \in \mathcal{T}_{k}$ there exists $t^{\prime \prime}>t^{\prime}$ such that $\gamma\left(t^{\prime \prime}\right) \in U$.

We remark that the (global) asymptotic behavior would not occur if just one of the reset maps were replaced by its inverse, for this situation, see Example 6 .

We stress that if solutions are not unique one distinguishes weak and strong local stability, as is the case for differential inclusions. For hybrid systems the introduction of reset maps makes the definition of stability more subtle, as equilibrium points can lie on the boundary of a polyhedral set. In this case, the examples identify two types of non-equivalent stable behavior: the local version where stability is confined to a single polyhedral set due to the "directions" of reset maps, and the global version where "directions" of reset maps do not play a role. The latter corresponds to the standard definition of stability (Goebel and Teel, 2006; Lygeros et al., 2003).

There are non-equilibrium points which possess properties similar to that of $x_{*}$ in Example 3 . For instance, define a hybrid system $(\mathcal{P}, \mathcal{S}, \mathcal{R})$ based on the data from Example 1 by $\mathcal{P}=K^{2}, \mathcal{S}=\mathcal{F}$, and $\mathcal{R}$ being a family of four identity maps such that $Q=\mathbb{R}^{2}$ can be identified with the quotient space $X / \sim$. We see that for any open neighborhood $U \subset X$ of $x \in O=\{(0 ; j) \mid j=1,2,3,4\}$ every trajectory $\left(\mathcal{T}_{k}, \gamma\right)$ will frequently belong to $U$, i.e., for any $t^{\prime} \in \mathcal{T}_{k}$ there exists $t^{\prime \prime}>t^{\prime}$ such that $\gamma\left(t^{\prime \prime}\right) \in U$.

Note that if $x \in O$ in the above is replaced by the four points making up $O$, i.e., $[(0 ; j)] \in X / \sim$ for any $j \in$ $\{1,2,3,4\}$, then we obtain a stronger stability property. More precise, for any open neighborhood $U \subset X$ of $O$, any trajectory $\left(\mathcal{T}_{k}, \gamma\right)$ will eventually belong to $U$, i.e., there exists $t^{\prime} \in \mathcal{T}_{k}$ such that $\gamma(t) \in U$ for $t \geq t^{\prime}$. Note that the set $O$ constitutes a single point in the quotient $X / \sim$, and it has the structure of an $\alpha$-limit set as known from the theory of ordinary differential equations.

The above illustration shows that a stability analysis must also include non-equilibrium points. Moreover, it indicates that such an analysis could be carried out in the quotient. This will be taken up in the next section, where we consider stability related to points which are not necessary equilibrium points.

4.2. Trajectories in the quotient. Any trajectory induces, in a natural way, a curve in the quotient space $X^{*}=X / \sim$. Formally, we have what follows.

Proposition 3. Any trajectory $\left(\mathcal{T}_{k}, \gamma\right)$ induces a continu- 
ous curve $\bar{\gamma}$ completing the diagram

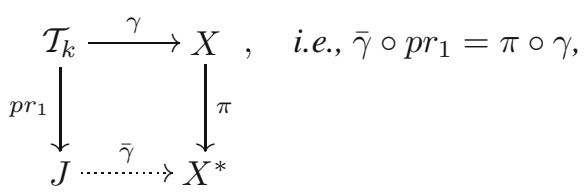

with $\mathrm{pr}_{1}$ as the projection on the first factor, and $J \subset$ $[0, \infty)$ its image. The curve $\bar{\gamma}$ will be called a quotient trajectory.

Two trajectories inducing the same quotient trajectory can be very different. For instance, their images may be contained in two different facets which are identified by $\sim$. However, we have the following "minimal" trajectory result with respect to number of discrete transitions.

Proposition 4. Let $\omega$ denote a quotient trajectory. Then there exists a minimal trajectory $\left(\mathcal{T}_{l}, \alpha\right)$ inducing $\omega$, that is, if $\left(\mathcal{T}_{k}, \gamma\right)$ is any trajectory inducing $\omega$ then $l \leq k$ and there is an injection $\iota: \mathcal{T}_{l} \rightarrow \mathcal{T}_{k}$ such that $\alpha=\gamma \circ \iota$.

Proof. Let $\left(\mathcal{T}_{k}, \gamma\right)$ be as above and define $L=$ $\left\{i \in \mathbf{k} \mid \operatorname{int}_{\mathbb{R}}\left(T_{i}\right) \neq \emptyset\right\}$. Note that $L$ is non-empty by Definition 2 Let $l$ be the cardinality of $L$, and $\nu: 1 \rightarrow L$ be the order preserving bijection. Note that $l \leq k$.

The trajectory $\left(\mathcal{T}_{l}, \alpha\right)$ is now defined by

$$
\mathcal{T}_{l}=\left\{\bigsqcup_{i \in \mathrm{l}} S_{i} \mid S_{i}=T_{\nu(i)} \subset \mathcal{T}_{k}\right\}
$$

and

$$
\alpha(t ; i)=\gamma(t, \nu(i))
$$

Now, if we let $\iota=\mathrm{id} \times \nu$, we obtain, by construction, a commutative diagram

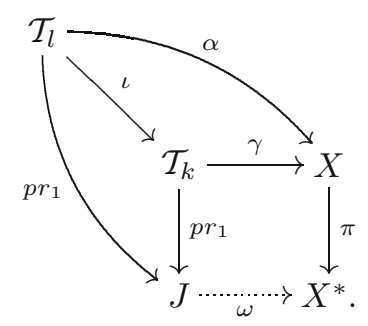

Thus $\alpha$ also induces $\omega$, hence completing the proof.

The proof above is constructive in the sense that, given any trajectory inducing $\omega$, Eqn. (3) constructs, out of this trajectory, a minimal trajectory inducing $\omega$.

4.2.1. Quotient stability. We are in a position to define concepts which relate stability to non-equilibrium points. A point $x \in X$ will be called quotient stable (resp. weakly quotient stable) if it is stable in the quotient, i.e., if for each open neighborhood $V \subset X^{*}$ of $\pi(x)=[x]$ there exists an open neighborhood $U \subset V$ of $\pi(x)$ such that for each $z \in U$ and for each (resp. some) quotient trajectory $\bar{\gamma}$ at $z$ we have $\bar{\gamma}(t) \in V$ for all $t \in \mathcal{T}_{k}$. A point which is not weakly quotient stable is called quotient unstable.

A point $x \in X$ is called asymptotically quotient stable (resp. weakly asymptotically quotient stable) if it is quotient stable (resp. weakly quotient stable) and $\bar{\gamma}(t) \rightarrow$ $\pi(x)$ for $t \rightarrow \infty$.

Example 2 shows that a locally (weakly) asymptotically stable equilibrium point can be quotient unstable. On the other hand, Example 3 shows that an asymptotically quotient stable equilibrium point can be locally unstable. Therefore, the two notions of stability do, in general, not imply each other. However, from the definition of the equivalence relation $\sim$, we immediately obtain the following proposition.

Proposition 5. Let $x_{*} \in \operatorname{int}_{\mathbb{R}^{n}}(P)$, for some $P \in X$, be an equilibrium. Then $x_{*}$ is locally asymptotically stable iff it is asymptotically quotient stable.

Moreover, if in Example 3 we replace one of the reset maps, say $R_{i}$, with its inverse, the point $\pi\left(x_{*}\right)$ remains quotient stable. The point $\pi\left(x_{*}\right)$ will not be asymptotically quotient stable since each quotient trajectory is induced by a trajectory and no trajectory has asymptotically stable behavior (each trajectory terminates on the domain of $R_{i}$, see also Example 6. This also illustrates that the change of a reset map can leave the topology of the quotient space unaltered, but at the same time alter the behavior of the quotient trajectories.

The following result gives a necessary and sufficient condition for an equilibrium point to be asymptotically quotient stable.

Proposition 6. Let $x_{*} \in X$ be an equilibrium. Then $x_{*}$ is (weakly) asymptotically quotient stable iff for each open neighborhood $V \subset X$ of the $\sim$-equivalence class $\left[x_{*}\right]$ there exists an open neighborhood $U \subset V$ of $\left[x_{*}\right]$ such that for each $x \in U$ and for each (resp. some) trajectory $\left(\mathcal{T}_{k}, \gamma\right)$ at $x$ we have $\gamma(t) \in V$ for all $t \in \mathcal{T}_{k}$.

Proof. We prove the "if" direction while the "only if" direction can be proven by similar arguments. Let $V^{\prime}$ be an open neighborhood of $\pi\left(x_{*}\right)$, and write $V=\pi^{-1}\left(V^{\prime}\right)$. Let $U \subset V$ be an open neighborhood of $\left[x_{*}\right]$ such that for any $x \in U$ and any (resp. some) trajectory $\left(\mathcal{T}_{k}, \gamma\right)$ at $x$, $\gamma(t) \in V$ for all $t \in \mathcal{T}_{k}$ and $\lim _{t \rightarrow \infty} \gamma(t)=x$.

Let $U^{\prime} \subset V^{\prime}$ be an open neighborhood of $\pi\left(x_{*}\right)$ such that $\pi^{-1}\left(U^{\prime}\right) \subset U$. Then for any $z \in U^{\prime}$ and any (resp. some) quotient trajectory $\bar{\gamma}$ at $z, \bar{\gamma}(t) \in V^{\prime}$ for all $t \in$ $[0, \infty)$. Otherwise, there would be a trajectory $\left(\mathcal{T}_{k}, \gamma\right)$ at $x \in \pi^{-1}(z) \subset U$, inducing $\bar{\gamma}$, and leaving $V$. Moreover, $\lim _{t \rightarrow \infty} \bar{\gamma}(t)=\pi\left(x_{*}\right)$ since, if not there would exist an open neighborhood $O$ of $\left[x_{*}\right]$ such that $\gamma(t) \notin O$ for all $t \in \mathcal{T}_{k}$, where $\left(\mathcal{T}_{k}, \gamma\right)$ is a trajectory at $x \in \pi^{-1}(z) \subset U$, inducing $\bar{\gamma}$. This proves the "if" direction. 
A more throughout analysis of quotient stability will be taken up in future works. Such an analysis will most likely be based on techniques similar to those of Blanchini and Miani (2008).

\section{Local analysis of hybrid systems}

Inspired by the theory of (differentiable) manifolds, we introduce the notion of a chart. This concept will enable a local analysis of hybrid systems and aid in the classification of "well behaved" hybrid systems such as regular and omnidirectional hybrid systems introduced in Section 5.1

Definition 3. (Chart) Let $\left(\mathcal{P}_{J}, \mathcal{S}, \mathcal{R}\right)$ be a hybrid system of dimension $n$, and $(U, Q, \psi)$ a triple where $U \subset X=$ $\bigsqcup_{j \in J} P_{j}$ has a non-empty interior, and $\left\{U \cap P_{j}\right\}_{j \in J}$ is a family of polyhedral sets, $Q \subset \mathbb{R}^{n}$ is a polyhedral set, and $\psi: U \rightarrow Q$ is a continuous surjection. The triple $(U, Q, \psi)$ will be called a chart on $\left(\mathcal{P}_{J}, \mathcal{S}, \mathcal{R}\right)$ if

(i) for any $x, y \in U, \psi(x)=\psi(y)$ if and only if $x \sim y$;

(ii) for any $P \in \mathcal{P}$ with $P \cap U \neq \emptyset$, the restriction $\left.\psi\right|_{U \cap P}$ is injective and the restriction of a linear map $\mathbb{R}^{n} \rightarrow$ $\mathbb{R}^{n}$;

(iii) if $x \in U$, then $[x] \subset U$, where $[x]$ denotes the $\sim$-lence class containing $x$.

A quasi chart is a chart fulfilling conditions (i) and (ii).

In general, the linear extension of $\left.\psi\right|_{U \cap P}$ from condition (ii) above is one among many linear extensions of $\left.\psi\right|_{U \cap P}$. However, if $\operatorname{int}_{\mathbb{R}^{n}}(U \cap P) \neq \emptyset$, then the linear extension is unique and injective.

To reduce technicalities in the exposition, we have chosen to formulate condition (ii) with a linear extension of $\left.\psi\right|_{U \cap P}$; nevertheless, the linear extension can be substituted by an affine extension, and all the results presented in this paper are still valid.

The basic properties of charts will now be presented.

Proposition 7. Let $(U, Q, \psi)$ denote a quasi chart on a hybrid system $(\mathcal{P}, \mathcal{S}, \mathcal{R})$ of dimension $n$. Then $Q$ has dimension $n$.

Proof. On the outset, let us remark that, since $U$ has a non-empty interior in $X$, there exists at least one $P \in \mathcal{P}$ such that $\operatorname{int}_{\mathbb{R}^{n}}(P \cap U) \neq \emptyset$. Let $P$ denote such an element of $\mathcal{P}$, and choose $x \in \operatorname{int}_{\mathbb{R}^{n}}(P \cap U)$. Hence, there exists an open neighborhood $U_{x} \subset \mathbb{R}^{n}$ of $x$ such that $U_{x} \subset$ $(P \cap U)$. Since $\left.\psi\right|_{U \cap P}$ is continuous and injective, we may apply Brouwer's theorem on invariance of domain to the map $\psi: U_{x} \rightarrow \mathbb{R}^{n}$, and conclude that $\psi\left(U_{x}\right)$ is open in $\mathbb{R}^{n}$. This concludes the proof since $\psi\left(U_{x}\right) \subset Q$.

Proposition 8. Let $(U, Q, \psi)$ denote a quasi chart on a hybrid system $(\mathcal{P}, \mathcal{S}, \mathcal{R})$ of dimension $n$. Then $\psi$ is a quotient map.
Proof. By continuity, $\psi^{-1}(V) \subset U$ is open whenever $V \subset Q$ is open. Hence, we need to prove that $V$ is open whenever $\psi^{-1}(V)$ is open.

Let $z \in V$ and $x \in \psi^{-1}(z)$. Therefore, $x \in P$ for some $P \in \mathcal{P}$. Since $\psi^{-1}(V)$ is open in $U$, we have by definition that $\psi^{-1}(V) \cap P \cap U$ is open in $P \cap U$. Hence, there exists an open neighborhood $B_{x} \subset \mathbb{R}^{n}$ of $x$ such that $\left(B_{x} \cap P \cap U\right) \subset\left(\psi^{-1}(V) \cap U \cap P\right)$. Now, let $\Psi: \mathbb{R}^{n} \rightarrow \mathbb{R}^{n}$ denote any continuous injective extension of $\left.\psi\right|_{U \cap P}$, e.g., a linear injective extension. By the invariance of domain (or the open mapping theorem in the linear case), we conclude that $\Psi\left(B_{x}\right)$ is open in $\mathbb{R}^{n}$. In particular, $Q \cap \Psi\left(B_{x}\right)$ is an open neighborhood of $z$ in $Q$, which is contained in $V$ since

$Q \cap \Psi\left(B_{x}\right)=\Psi(U \cap P) \cap \Psi\left(B_{x}\right)=\Psi\left(U \cap P \cap B_{x}\right)$,

and

$$
\Psi\left(U \cap P \cap B_{x}\right) \subset \Psi\left(\psi^{-1}(V) \cap U \cap P\right) \subset V .
$$

Hence, $z$ is interior to $V$ proving that $V$ is open since $z$ was arbitrary.

Lemma 2. Let $(U, Q, \psi)$ denote a quasi chart on a hybrid $\operatorname{system}(\mathcal{P}, \mathcal{S}, \mathcal{R})$ and $U^{*}=U / \sim$ the quotient space. The map $\psi$ induces a map g completing the diagram

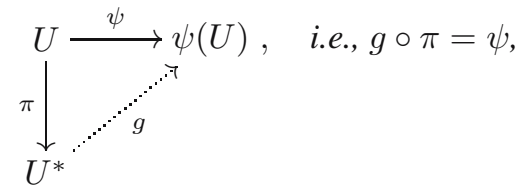

with $\pi$ as the canonical map. Furthermore, $g$ is a homeomorphism.

Proof. By condition (i) of Definition 3, we have $U^{*}=$ $\left\{\psi^{-1}(v) \mid v \in \psi(U)\right\}$. Hence, $g$ is a homeomorphism by Munkres (1975, Corollary 22.3).

Standing hypothesis. The following will be in force throughout the rest of this paper. For any hybrid system $(\mathcal{P}, \mathcal{S}, \mathcal{R})$ the family $\mathcal{P}$ is assumed to consist of polytopes only.

Requiring that $\mathcal{P}$ consist of polytopes only is a consequence of the implicit use of Lemma A1 (via Lemma A5 in the proof of Theorem 1 below. However, we do conjecture that this requirement can be dropped without effecting the conclusion of the theorem (and therefore that the hypotheses can be removed altogether).

The next result shows that locally a chart can be used to make a hybrid system resemble a switched system.

Theorem 1. Let $(U, Q, \psi)$ be a chart on a hybrid system $\left(\mathcal{P}_{J}, \mathcal{S}, \mathcal{R}\right)$, and

$$
J(U)=\left\{j \in J \mid \operatorname{int}_{\mathbb{R}^{n}}\left(P_{j} \cap U\right) \neq \emptyset, P_{j} \in \mathcal{P}\right\} .
$$


Let $Q_{j}=\psi\left(U \cap P_{j}\right), K^{n}=\left\{Q_{j} \mid j \in J(U)\right\}$ and $K=\left\{F \mid F \preceq Q_{j}, Q_{j} \in K^{n}\right\}$. Let $\psi_{j}=\left.\psi\right|_{U \cap P_{j}}$, $f_{j}=\mathrm{d} \psi_{j} \xi_{j} \circ \psi_{j}^{-1}: Q_{j} \rightarrow \mathbb{R}^{n}$ and $\mathcal{F}=\left\{f_{j} \mid j \in J(U)\right\}$. Then $(Q, K, \mathcal{F})$ is a switched system.

Before beginning the proof, we remark that the differential $\mathrm{d} \psi_{\mathrm{j}}$ is indeed well defined since $j \in J(U)$ and $\psi_{j}$ has a unique (linear) extension.

Proof. (Proof of Theorem 11) By Definition [3, we immediately conclude that $Q_{j}$ is a polytope; hence, $K$ (and $K^{n}$ ) is a family of polytopes (of dimension $n$ ). To prove the theorem, we need to show that $K$ is a partition of $Q$ (see Section 2.1.1).

We prove that $K$ is a complex. Let $F_{1}, F_{2} \in K$. Since $F_{i} \in K, i=1,2$, there is $Q_{i} \in K^{n}$ such that $F_{i} \preceq Q_{i}$. By Lemma A5, $Q_{1} \cap Q_{2}$ is a face of $Q_{1}$ and $Q_{2}$. By Lemma $F_{1} \cap F_{2} \preceq Q_{1} \cap Q_{2}$. So $F_{1} \cap F_{2}$ is a face of both $Q_{1}$ and $Q_{2}$, thus also of $F_{1}$ and $F_{2}$. This proves property (ii). Therefore, $K$ is a complex since property (i) is trivial.

We will show that $Q=\bigcup_{j \in J(U)} Q_{j}$; hence, $Q=$ $|K|$, which will complete the proof. For any point $x \in$ $Q$ and any open neighborhood $V$ of $x$ in $Q, \psi^{-1}(V)$ is open in $U$. Hence, there is $j \in J$ such that $P_{j} \cap \psi^{-1}(V)$ has a non-empty interior, thus $j \in J(U)$ and $x \in Q_{j}$. Therefore, $Q \subset \bigcup_{j \in J(U)} Q_{j}$, which completes the proof since clearly $\bigcup_{j \in J(U)} Q_{j} \subset \bigcup_{j \in J} Q_{j}=Q$.

The construction in Theorem 1 gives rise to the following definition.

Definition 4. Let $(U, Q, \psi)$ be a chart on a hybrid system $\left(\mathcal{P}_{J}, \mathcal{S}, \mathcal{R}\right)$. The switched system $(Q, K, \mathcal{F})$ defined in Theorem 1 will be called a switched system (with state-dependent switching) generated by the chart $(U, Q, \psi)$

A switched system generated by a chart is obtained by "gluing" together (images of) subsets from a hybrid system. A local representation of a hybrid system is particularly nice in that it always produces a polytope. Therefore, more complex structures will be absent in a chart. More precisely, there are hybrid systems containing open sets which can not be represented in any chart. We illustrate this in the next example.

Example 4. Let $\left(\mathcal{P}_{J}, \mathcal{S}, \mathcal{R}\right)$ with $J=\{1,2,3\}$ be a hybrid system of dimension 1 , where $\mathcal{S}$ is left unspecified, $\mathcal{P}=\left\{P_{i}=[0,1]\right\}_{i \in J}$, and $\mathcal{R}=\left\{R_{1}: F_{1} \rightarrow F_{2}, R_{2}:\right.$ $\left.F_{2} \rightarrow F_{3}, R_{3}: F_{3} \rightarrow F_{1}\right\}$ with $F_{i}, i \in J$, being a facet of $P_{i}$. Hence $X^{*}$ has a "Y" shape. Therefore, if $p \in F_{i}$, no open neighborhood of $[p]$ in $X$ can be contained in a chart.

As seen above, hybrid systems can contain structures which cannot be represented in a chart. For a global analysis this property is highly undesirable. Therefore, a class of hybrid systems is introduced, whose structure is well behaved in the sense that for any point there exists a chart which gives a correct local representation of some open neighborhood of that point. To this end, we define the notion of a regular hybrid system.

Definition 5. (Regular hybrid system) A hybrid system $\left(\mathcal{P}_{J}, \mathcal{S}, \mathcal{R}\right)$ is regular if there is a family of charts $\left\{\left(U_{a}, Q_{a}, \psi_{a}\right) \mid a \in A\right\}$ such that

(i) $\pi\left(V_{a}\right)$, with $V_{a}=\psi_{a}^{-1}\left(\operatorname{int}\left(Q_{a}\right)\right)$, is open in $X^{*}$ for all $a \in A$

(ii) $X=\bigcup_{a \in A} V_{a}$.

A chart fulfilling (i) above will be called regular, and if $x \in V_{a}$ then $\left(U_{a}, Q_{a}, \psi_{a}\right)$ will be called a regular chart at $x$.

The next example illustrates the concept of a regular hybrid system.

Example 5. Let $\left(\mathcal{P}_{J}, \mathcal{S}, \mathcal{R}\right)$ with $J=\{1,2\}$ be a hybrid system of dimension 2, where $\mathcal{S}$ is left unspecified, $\mathcal{P}=$ $\left\{P_{i}=I^{2}\right\}_{i \in J}$ with $I=[0,1]$, and $\mathcal{R}=\left\{R_{i}=i d\right.$ : $\left.F_{1}^{i} \rightarrow F_{2}^{i}\right\}_{i \in J}$ with $F_{i}^{1}=I \times\{0\} \prec P_{i}, i \in J$, and $F_{i}^{2}=I \times\{1\} \prec P_{i}, i \in J$. Hence $X^{*}$ is a "cylinder".

The hybrid system $(\mathcal{P}, \mathcal{S}, \mathcal{R})$ becomes regular by introducing the following two charts: $\left(U_{1}, Q_{1}, \psi_{1}\right)$ and $\left(U_{2}, Q_{2}, \psi_{2}\right)$, where

$$
\begin{aligned}
U_{1} & =I \times[0,0.75] \times\{1\} \cup I \times[0,0.75] \times\{2\}, \\
U_{2} & =I \times[0.25,1] \times\{1\} \cup I \times[0.25,1] \times\{2\}, \\
Q_{1} & =I \times[-0.75,0.75], \\
Q_{2} & =I \times[0.25,1.75],
\end{aligned}
$$

and

$$
\begin{array}{ll}
\psi_{1}(x, y ; 1) & =(x, y), \quad \psi_{1}(x, y, 2)=(x,-y), \\
\psi_{2}(x, y ; 1)=(x, y), \quad \psi_{2}(x, y ; 2)=(x, 2-y) .
\end{array}
$$

A regular hybrid system of dimension $n$ induces the structure of a topological $n$-manifold (Lang, 1999) on $X^{*}$ via the family of charts $\left\{\left(\pi\left(V_{a}\right), g_{a}\right)\right\}_{a \in A}$, with $g_{a}$ being the map induced by $\psi_{a}$ as in Lemma 2. In particular, for a regular hybrid system, $\left(X^{*},\left\{\left(\pi\left(V_{a}\right), g_{a}\right)\right\}_{a \in A}\right)$ is a PL space as defined by Hudson (1969), and if the vector fields $f_{i}$ all are linear we obtain a PL system as in the works of Sontag (1981) as well as Rantzer and Johansson (2000). Note that the hybrid system in Example 4 is not regular since $X^{*}$ is not a topological manifold.

5.1. Omnidirectional hybrid systems. Up to now, we have been concerned with how to obtain a precise local representation of the structure (or geometry) of a hybrid system, and ended with the concept of a regular hybrid 
system. A natural question is now whether or not locally the dynamics of a hybrid system can be represented by those of a switched system generated by charts. As we have seen above, this may fail for general hybrid systems since there can be an open neighborhood that has no local representation.

The next proposition states that each trajectory of a hybrid system which is contained in a chart is related to a trajectory of a switched system generated by this chart.

Proposition 9. Let $(U, Q, \psi)$ be a chart on a hybrid system $\left(\mathcal{P}_{J}, \mathcal{S}, \mathcal{R}_{L}\right)$ and $(Q, K, \mathcal{F})$ be a switched system generated by $(U, Q, \psi)$. If $\left(\mathcal{T}_{k}, \gamma\right)$ is a trajectory of $\left(\mathcal{P}_{J}, \mathcal{S}, \mathcal{R}\right)$ such that $\gamma(t ; i) \in U$ for all $(t ; i) \in \mathcal{T}_{k}$, then

$$
\eta(t)=\psi(\gamma(t ; i))
$$

is a solution of the Cauchy problem $\dot{\eta} \in F(\eta), \eta\left(t_{0}\right)=$ $\psi\left(\gamma\left(t_{0} ; 1\right)\right)$, where $F: Q \rightarrow 2^{\mathbb{R}^{n}}$ is given by

$$
\begin{aligned}
& F(x)=\left\{v \in \mathbb{R}^{n} \mid v=f_{j}(x)\right. \\
& \left.\quad \text { if } x \in Q_{j} \text { for } j \in J(U), f_{j} \in \mathcal{F}\right\}
\end{aligned}
$$

with $J(U)$ as in (4).

Before beginning the proof, let us remark that the proposition would be false if in the definition of a trajectory (Definition 2) the requirement int $\mathbb{R}_{\mathbb{R}}\left(T_{i}\right) \neq \emptyset$ had been excluded. Indeed, if $\operatorname{int}_{\mathbb{R}}\left(T_{i}\right)=\emptyset$ for all $i \in \mathbf{k}$, then the induced trajectory (5) would have a singleton set as the domain, but any solution to $\dot{\eta} \in F(\eta)$ is by definition required to have a domain with a non-empty interior.

Proof. (Proof of Proposition 9) Let $\left\{t_{i}\right\}_{i \in \mathbf{k}_{0}}$ be the switching sequence corresponding to $\mathcal{T}_{k}$. For each $i \in \mathbf{k}$ there exists $j \in J(U)$ such that $\dot{\gamma}(t ; i)=\xi_{j}(\gamma(t ; i))$ for all $t \in\left[t_{i-1}, t_{i}\right]$.

The vector fields $f_{j} \in \mathcal{F}$ and $\xi_{j} \in \mathcal{S}$ are $\psi_{j}$-related. Thus, $\gamma$ and the solution $\eta$ of the Cauchy problem $\dot{\eta}(t)=f_{j}(\eta(t)), \eta\left(t_{i-1}\right)=\psi\left(\gamma\left(t_{i-1} ; i\right)\right)$ commute in the following sense: $\psi(\gamma(t ; i))=\eta(t)$ for $t \in\left[t_{i-1}, t_{i}\right]$.

This completes the proof since $\psi\left(\gamma\left(t_{i} ; i\right)\right)=$ $\psi\left(\gamma\left(t_{i} ; i+1\right)\right)$ by point (iii) in Definition 2

There are hybrid systems for which there exists a solution of $\dot{\eta} \in F(\eta)$, but there is no trajectory $\left(\mathcal{T}_{k}, \gamma\right)$ such that $\eta$ and $\gamma$ are related by (5). This is the context of the next example.

Example 6. Let $C=[-r, r]^{2}$ for some $r>0$. Consider the hybrid system from Example 3 with each $P_{i}$ replaced by $P_{i}^{\prime}=P_{i} \cap C$, and the reset map $R_{1}$ replaced by $R_{1}^{-1}$, i.e., $\mathcal{R}=\left\{R_{1}^{-1}, R_{2}, R_{3}, R_{4}\right\}$. We use the chart $\left(C^{\prime}, C, \psi\right)$, where $C^{\prime}=\bigsqcup P_{i}^{\prime}$ and $\psi \mid P_{i}^{\prime}=$ id. Any trajectory of the hybrid system is defined on a bounded time domain; it terminates on the boundary of $P_{1}$. However, the solutions of $\dot{\eta} \in F(\eta)$ are defined on $[0, \infty)$ and exhibit an asymptotically stable behavior (in $C)$.
Now, if, in Example $6 \mathcal{R}=\left\{R_{i}, R_{i}^{-1} \mid i=1, \ldots, 4\right\}$ had been used instead, then for any $\eta$ there is a $\gamma$ such that (5) holds. This leads to the following definition.

Definition 6. (Omnidirectional hybrid system) A hybrid system $\left(\mathcal{P}_{J}, \mathcal{S}, \mathcal{R}\right)$ is omnidirectional if $\mathcal{R}=\mathcal{R}^{0}$.

For omnidirectional hybrid systems we have the following converse to Proposition 9 .

Proposition 10. Let $(U, Q, \psi)$ be a chart on a omnidirectional hybrid system $\left(\mathcal{P}_{J}, \mathcal{S}, \mathcal{R}_{L}\right)$ and $(Q, K, \mathcal{F})$ be a switched system generated by $(U, Q, \psi)$. Then for any solution $\eta$ of $\dot{\eta} \in F(\eta)$ with $F$ defined by (6) there is a trajectory $\left(\mathcal{T}_{k}, \gamma\right)$ of $\left(\mathcal{P}_{J}, \mathcal{S}, \mathcal{R}_{L}\right)$ such that (5) holds.

Proof. Let $\eta$ satisfy $\dot{\eta}(t) \in F(\eta(t))$ a.e. on $[0, T]$, with $0<T<\infty$, and define $j(t) \in J$ for almost all $t \in[0, T]$ by

$$
j(t)=j \quad \text { iff } \quad \dot{\eta}(t)=\mathrm{d} \psi_{j} \xi_{j} \circ \psi_{j}^{-1}(\eta(t)),
$$

with the notation as in Theorem 1. Let $\left\{t_{i}\right\}_{i \in \mathbf{k}_{0}}$, with $k \in \mathbb{N} \cup\{\infty\}$, be an increasing sequence of points in $[0, T]$, where $j(t)$ is undefined. Hence, $t_{0}=0, t_{k}=T$, and $j(t)$ is constant on $I_{i}=\left(t_{i-1}, t_{i}\right)$ for $i \in \mathbf{k}$. We assume, without loss of generality, that $j(\cdot)$ is not constant on each open neighborhood of each $t_{i}$. Moreover, let $j_{i}=$ $j_{i}(t)$ denote the obvious extension of (the constant) $j(t)$ to $\mathrm{cl}\left(I_{i}\right)$

Now, for each $i \in \mathbf{k}$, define the map $\gamma_{i}$ on $I_{i}$ by $\gamma_{i}(t)=\psi_{j(t)}^{-1} \circ \eta(t)$ and extend it to a continuous map on $\operatorname{cl}\left(I_{i}\right)$. Hence $\gamma_{i}$ is differentiable on $I_{i}$ with the derivative

$$
\dot{\gamma}_{i}(t)=\xi_{j(t)}\left(\gamma_{i}(t)\right)
$$

The trajectory $\left(\mathcal{T}_{k}, \gamma\right)$ is now defined by $\mathcal{T}_{k}=$ $\bigsqcup_{i \in \mathbf{k}} \operatorname{cl}\left(I_{i}\right)$ and $\gamma(t ; i)=\left(\gamma_{i}(t) ; j_{i}\right)$. Indeed, since $0<T$, we must have $\operatorname{int}\left(T_{i}\right) \neq \emptyset$ for at least one $i \in \mathbf{k}$; hence, $\mathcal{T}_{k}$ is a time domain. Moreover, $\gamma: \mathcal{T}_{k} \rightarrow \bigsqcup_{j \in J} P_{j}$ is continuous by construction, and, to complete the proof, points (i)-(iii) of Definition 2 need to be verified. To this end, point (iii) is a consequence of (7), and points (i) and (iii) follow from Definition 3 as $j_{i} \neq j_{i+1}$ for each $i \in \mathbf{k}^{*}$.

For omnidirectional hybrid systems there is a local correspondence between trajectories of the system and trajectories generated by charts. For this observation to have any significance in stability analysis, we need to verify that the behavior of these two systems agrees. Indeed, the next theorem provides necessary and sufficient conditions for an equilibrium point of an omnidirectional hybrid system $(\mathcal{P}, \mathcal{S}, \mathcal{R})$ to be asymptotically quotient stable in terms of conditions imposed on a switched system generated by a chart on $(\mathcal{P}, \mathcal{S}, \mathcal{R})$.

For the next theorem, recall the notion of $G$-stability as described below Proposition 1 in Section 3 . 
Theorem 2. Let $\left(\mathcal{P}_{J}, \mathcal{S}, \mathcal{R}\right)$ be a regular omnidirectional hybrid system. Then the equilibrium $x_{*} \in X$ is (weakly) asymptotically quotient stable iff $\psi\left(x_{*}\right)$ is a (weakly) asymptotically $F$-stable equilibrium, where $(U, Q, \psi)$ is a regular chart at $x_{*}$, and $F$ is defined by (6).

Proof. We prove the "only if" direction. Since $\left(\mathcal{P}_{J}, \mathcal{S}, \mathcal{R}\right)$ is regular, a regular chart $(U, Q, \psi)$ at $x$ exists. Let $O$ be an open neighborhood of $\psi\left(x_{*}\right)$ in $\operatorname{int}(Q)$, and let $W=\psi^{-1}(O)$. Since the equilibrium $x_{*}$ is (weakly) asymptotically quotient stable, we use Proposition 6 to conclude that there is an open neighborhood $W^{\prime} \subset W$ of $\left[x_{*}\right]$ such that for each $x \in W^{\prime}$ and for each (resp. some) trajectory $\left(\mathcal{T}_{k}, \gamma\right)$ at $x$ we have $\gamma(t, i) \in W$ for all $(t, i) \in \mathcal{T}_{k}$ and $\gamma(t, i) \rightarrow x_{*}$ as $t \rightarrow \infty$.

Let $O^{\prime} \subset O$ be an open neighborhood of $\psi\left(x_{*}\right)$ such that $\psi^{-1}\left(O^{\prime}\right) \subset W^{\prime}$. By Proposition 10 , we conclude that for any (respectively some) solution $\eta$ of $\dot{\eta} \in F(\eta)$ at $y \in O^{\prime}$ we have $\eta(t) \in O$ for all $t \geq 0$, and $\eta(t) \rightarrow \psi\left(x_{*}\right)$ as $t \rightarrow \infty$.

The proof of the converse is analogous, with Proposition 9 used instead of Proposition 10

The aim of our work was to restrict the definition of hybrid systems in such a way that development of tangible methods for stability is feasible. In conclusion, we have defined a locally switched system, which is a relatively restrictive hybrid system, but allows analyzing using standard methods of dynamical systems and, in particular, differential inclusions. That is, Theorem 2 together with Proposition 2 may be used to conduct a stability analysis as described in Section 3 . Nevertheless, some care should be taken when using Proposition 2 as it deals with relaxed solutions.

\section{Conclusion}

We have introduced various notions of stability for a hybrid system on a polyhedral set with state-dependent switching. In particular, the concept of stability has been extended to non-equilibrium points via a quotient system. It has been shown that for such systems an equilibrium is asymptotically stable if and only if it is asymptotically stable in the quotient system. Moreover, necessary and sufficient conditions have been provided for an equilibrium point of a regular omnidirectional hybrid system to be asymptotically stable. These conditions have been formulated in terms of a switched system (with state-dependent switching) generated by a chart on the system. As methods for stability analysis of switched systems are already available, this work establishes tangible means for stability analysis of hybrid systems.

\section{References}

Ames, A. and Sastry, S. (2005). A homology theory for hybrid systems: Hybrid homology, in M. Morari and L. Thiele
(Eds.), Hybrid Systems: Computation and Control, Lecture Notes in Computer Science, Vol. 3414, Springer-Verlag, Berlin/Heidelberg, pp. 86-102.

Balluchi, A., Benvenuti, L. and Sangiovanni-Vincentelli, A. (2005). Hybrid systems in automotive electronics design, 44th IEEE Conference on Decision and Control \& 2005/2005 European Control Conference, CDC-ECC '05, Seville, Spain, pp. 5618-5623.

Blanchini, F. and Miani, S. (2008). Set-theoretic Methods in Control, Systems \& Control: Foundations \& Applications, Birkhäuser, Boston, MA.

Bredon, G.E. (1993). Topology and Geometry, Graduate Texts in Mathematics, Vol. 139, Springer-Verlag, New York, NY.

Bujorianu, M.L. and Lygeros, J. (2006). Toward a general theory of stochastic hybrid systems, in $\mathrm{H}$. Bloom and J. Lygeros (Eds.), Stochastic Hybrid Systems, Lecture Notes in Control and Information Sciences, Vol. 337, Springer, Berlin, pp. 3-30.

Ding, J., Gillulay, J.H., Huang, H., Vitus, M.P., Zhang, W. and Tomlin, C. (2011). Hybrid systems in robotics, IEEE Robotics \& Automation Magazine 18(3): 33-43.

Goebel, R., Sanfelice, R.G. and Teel, A.R. (2009). Hybrid dynamical systems: Robust stability and control for systems that combine continuous-time and discrete-time dynamics, IEEE Control Systems Magazine 29(2): 28-93.

Goebel, R. and Teel, A. R. (2006). Solutions to hybrid inclusions via set and graphical convergence with stability theory applications, Automatica 42(4): 573-587.

Grünbaum, B. (2003). Convex Polytopes, 2nd Edn., Graduate Texts in Mathematics, Vol. 221, Springer-Verlag, New York, NY.

Habets, L.C.G.J.M. and van Schuppen J.H. (2005). Control to facet problems for affine systems on simplices and polytopes-with applications to control of hybrid systems, Proceedings of the 44th IEEE Conference on Decision and Control, Seville, Spain, pp. 4175-4180.

Haddad, W.M., Chellaboina, V. and Nersesov, S.G. (2006). Impulsive and Hybrid Dynamical Systems, Princeton Series in Applied Mathematics, Princeton University Press, Princeton, NJ.

Heemels, W.P.M.H., De Schutter, B. and Bemporad, A. (2001). Equivalence of hybrid dynamical models, Automatica 37(7): 1085-1091.

Hudson, J.F.P. (1969). Piecewise Linear Topology, University of Chicago Lecture Notes, W.A. Benjamin, Inc., New York, NY/Amsterdam.

Johansson, M. and Rantzer, A. (1998). Computation of piecewise quadratic Lyapunov functions for hybrid systems, IEEE Transactions on Automatic Control 43(4): 555-559.

Kunze, M. (2000). Non-smooth Dynamical Systems, Lecture Notes in Mathematics, Vol. 1744, Springer-Verlag, Berlin.

Lang, S. (1999). Fundamentals of Differential Geometry, Graduate Texts in Mathematics, Vol. 191, Springer-Verlag, New York, NY. 
Leine, R.I. and Nijmeijer, H. (2004). Dynamics and Bifurcations of Non-smooth Mechanical Systems, Lecture Notes in Applied and Computational Mechanics, Vol. 18, Springer-Verlag, Berlin.

Leth, J. and Wisniewski, R. (2012). On formalism and stability of switched systems, Journal of Control Theory and Applications 10(2): 176-183.

Liberzon, D. (2003). Switching in Systems and Control, Systems \& Control: Foundations \& Applications, Birkhäuser, Boston, MA.

Lygeros, J., Johansson, K.H., Simić, S.N., Zhang, J. and Sastry, S.S. (2003). Dynamical properties of hybrid automata, IEEE Transactions on Automatic Control 48(1): 2-17.

Munkres, J.R. (1975). Topology: A First Course, Prentice-Hall, Englewood Cliffs, NJ.

Pettersson, S. and Lennartson, B. (2002). Hybrid system stability and robustness verification using linear matrix inequalities, International Journal of Control 75(16): 1335-1355.

Rantzer, A. and Johansson, M. (2000). Piecewise linear quadratic optimal control, IEEE Transactions on Automatic Control 45(4): 629-637.

Rienmüller, T., Hofbaur, M., Travé-Massuyès, L. and Bayoudh, M. (2013). Mode set focused hybrid estimation, International Journal of Applied Mathematics and Computer Science 23(1): 131-144, DOI: 10.2478/amcs-2013-0011.

Simić, S.N., Johansson, K.H., Lygeros, J. and Sastry, S. (2005). Towards a geometric theory of hybrid systems, Dynamics of Continuous, Discrete \& Impulsive Systems B: Applications \& Algorithms 12(5-6): 649-687.

Sontag, E.D. (1981). Nonlinear regulation: The piecewise linear approach, IEEE Transactions on Automatic Control 26(2): 346-358.

Tabuada, P. (2009). Verification and Control of Hybrid Systems, Springer, New York, NY.

Tomlin, C., Pappas, G.J. and Sastry, S. (1998). Conflict resolution for air traffic management: A study in multiagent hybrid systems, IEEE Transactions on Automatic Control 43(4): 509-521.

van der Schaft, A. and Schumacher, H. (2000). An Introduction to Hybrid Dynamical Systems, Lecture Notes in Control and Information Sciences, Vol. 251, Springer-Verlag, London.

Wisniewski, R. (2006). Towards modelling of hybrid systems, 45th IEEE Conference on Decision and Control, San Diego, CA, USA, pp. 911-916.

Wisniewski, R. and Leth, J. (2011). Convenient model for systems with hystereses-control, Proceedings of the 50th IEEE Conference on Decision and Control, Orlando, FL, USA.

Yang, H., Jiang, B., Cocquempot, V. and Chen, M. (2013). Spacecraft formation stabilization and fault tolerance: A state-varying switched system approach, System \& Control Letters 62(9): 715-722.
Yang, H., Jiang, B., Cocquempot, V. and Zang, H. (2011) Stabilization of switched nonlinear systems with all unstable modes: Application to multi-agent systems, IEEE Transactions on Automatic Control 56(9): 2230-2235.

Yordanov, B., Tůmová, J., Černá, I., Barnat, J. and Belta, C. (2012). Temporal logic control of discrete-time piecewise affine systems, IEEE Transactions on Automatic Control 57(6): 1491-1504.

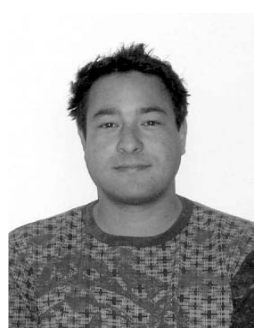

John Leth received his M.Sc. (2003) and Ph.D. (2007) degrees from the Department of Mathematical Sciences, Aalborg University, Denmark. Currently, he is employed as an assistant professor at the Department of Electronic Systems, Aalborg University. His research interests include mathematical control theory and (stochastic) hybrid systems.

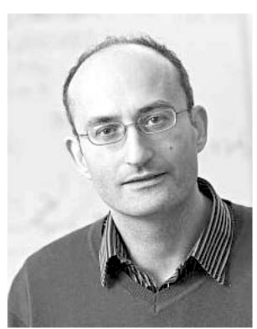

Rafael Wisniewski is a professor in the Section of Automation \& Control, Department of Electronic Systems, Aalborg University. He received a Ph.D. in electrical engineering in 1997, and a Ph.D. in mathematics in 2005. In 2007-2008, he was a control specialist at Danfoss A/S. His research interest is in system theory, particularly in hybrid systems.

\section{Appendix}

A1. Notation and terminology. If $S \subset \mathbb{R}^{n}$, then $\operatorname{aff}(S)$ denotes the affine hull (or span) of $S$ and by the dimension, $\operatorname{dim}(S)$, of $S$ we mean the affine dimension of $\operatorname{aff}(S)$, i.e., $\operatorname{dim}(S)=\operatorname{afdim}(\operatorname{aff}(S))=\operatorname{dim}(\operatorname{aff}(S) \ominus$ $\operatorname{aff}(S))$, where, in generic notation, $A \ominus B=\{a-b \mid a \in$ $A, b \in B\}$ with $A$ and $B$ being affine subspaces.

Let $N \in \mathbb{R}^{n}, \alpha \in \mathbb{R}$ and $\langle\cdot, \cdot\rangle$ denote an inner product in $\mathbb{R}^{n}$. We write $H(N, \alpha)=\left\{x \in \mathbb{R}^{n} \mid\langle x, N\rangle=\alpha\right\}$, $H^{+}(N, \alpha)=\left\{x \in \mathbb{R}^{n} \mid\langle x, N\rangle \geq \alpha\right\}$ and $H^{-}(N, \alpha)=$ $\left\{x \in \mathbb{R}^{n} \mid\langle x, N\rangle \leq \alpha\right\}$. We occasionally suppress the notation and simply write $H, H^{+}$, and $H^{-}$.

A2. Polyhedral sets and polytopes A polyhedral set $P$ in $\mathbb{R}^{n}$ is the intersection of a family of closed half spaces. A polytope is a bounded polyhedral set. Whenever a polyhedral set $P$ in $\mathbb{R}^{n}$ is considered a topological space, it will be understood that $P$ carries the relative topology from $\mathbb{R}^{n}$. A subset $F$ of a polyhedral set $P$ is a face if either $F=\emptyset$ or $F=P$, or there exists a supporting hyperplane $H$ of $P$ such that $F=P \cap H$. Note that a face is again a polyhedral set. A face $F$ of dimension $n-1$ is called a facet, and a face of dimension 0 is called a vertex. We write $F \preceq P$ to indicate that $F$ is a face of $P$ and $F \prec P$ if and only if $F \preceq P$ and $F \neq P$. Note that the relation $\preceq$ is a partial order relation on the set $K(P)$ of all faces of $P$.

For a nonempty $U \subset P$ with $P$ being a polyhedral 
set, we say $F \preceq P$ is the smallest face containing $U$ if $U \subset F$, and there is no other face $G$ of $P$ such that $U \subset G$ and $G \prec F$. Such a face always exists. Indeed, let $V=\{L \in K(P) \mid U \subset L\}$, with $K(P)$ as above, which is finite since $K(P)$ is finite, and non-empty since $P \in V$. Now, $F=\bigcap_{L \in V} L$ is a face of any $L \in V$, since the intersection of a finite number of faces is a face. Furthermore, $U \subset F$. We claim that $F$ is the smallest face of $P$ containing $U$. Suppose that there is $G \prec F$ with $U \subset G$, then $G \in V$; hence, $F \preceq G$, which is a contradiction.

Let $P$ and $P^{\prime}$ be polyhedral sets. A map $f: P \rightarrow P^{\prime}$ is said to be polyhedral if it is a continuous injection and if for any $F \preceq P$ there is $F^{\prime} \preceq P^{\prime}$ with $\operatorname{dim}(F)=\operatorname{dim}\left(F^{\prime}\right)$ such that $f(F) \subset F^{\prime}$. Note that if $P$ is a polytope then $f$ : $P \rightarrow f(P)$ is a homeomorphism. We remark that to the authors' best knowledge the concept of a polyhedral map, as presented here, is new. Nonetheless, related definitions can be found in the works of Hudson (1969) and Sontag (1981).

The following lemma, which is crucial for many proofs in the main body of the text, lists some basic properties of polyhedral maps. Note that, as a consequence of the use of Lemma A2, the lemma is proven for polytopes only.

Lemma A1. Let $P$ and $P^{\prime}$ be polytopes and $f: P \rightarrow P^{\prime}$ be polyhedral.

(i) If $F \preceq P$, then $f(F) \preceq P^{\prime}$ and $\operatorname{dim}(F)=$ $\operatorname{dim}(f(F))$

(ii) If $\operatorname{dim}(P)=\operatorname{dim}\left(P^{\prime}\right)$, then $f(P)=P^{\prime}$.

(iii) $f^{-1}: f(P) \rightarrow P$ is polyhedral.

Proof. We prove point (i) by induction on the dimension $k$ of $F$. For $k=0$, i.e., $F$ is a vertex, point (i) clearly holds. Suppose now that $f(L) \preceq P^{\prime}$ for any face $L$ of $P$ with $\operatorname{dim}(L)=k$. Let $F \preceq P$ and $\operatorname{dim}(F)=k+1$. Since $f$ is polyhedral, there is $F^{\prime} \preceq P^{\prime}$ such that $f(F) \subset F^{\prime}$ and $\operatorname{dim}\left(F^{\prime}\right)=k+1$. We show that $f(F)=F^{\prime}$, which then proves point (i).

By induction hypothesis, $f(L) \prec P$; hence, $f(L) \prec$ $F^{\prime}$, for any $L \prec F$. Now, let $L_{i}, i=1, \ldots, l$ be all facets of $F, L_{i}^{\prime}=f\left(L_{i}\right)$, and $F^{\prime}=\bigcap_{j=1}^{m} H_{j}^{+}$, where $\left\{H_{j}^{+} \mid 1 \leq j \leq m\right\}$ is an irredundant family of half-spaces with supporting hyperplanes $H_{j}$. Hence, $m \geq l, L_{i}^{\prime}=$ $H_{i} \cap F^{\prime}, i=1, \ldots, l$, after a possible re-indexing, and $\operatorname{bd}(f(F))=f(\operatorname{bd}(F))=\bigcup_{i=1}^{l} f\left(L_{i}\right)=\bigcup_{i=1}^{l} L_{i}^{\prime}$, where the first equality follows by Lemma A2

The hyperplane $H_{i}, i=1, \ldots, l$, is a supporting hyperplane of $f(F)$, since $f(F) \subset F^{\prime} \subset H_{i}^{+}$, and $L_{i}^{\prime}=H_{i} \cap f(F)$ as $L_{i}^{\prime} \subset H_{i} \cap f(F) \subset H_{i} \cap F^{\prime}=L_{i}^{\prime}$. Therefore, we conclude that

$$
F^{\prime}=\bigcap_{j=1}^{m} H_{j}^{+} \subset \bigcap_{j=1}^{l} H_{j}^{+}=f(F) .
$$

Hence, $f(F)=F^{\prime}$, proving point (i).

From point (i), we conclude that $f(P)$ is a face of $P^{\prime}$ with $\operatorname{dim}(f(P))=\operatorname{dim}(P)$. This proves point (ii).

First, $f(P)$ is a polyhedral set by point (i). Secondly, $f^{-1}: f(P) \rightarrow P$ is a continuous injection since $f: P \longrightarrow$ $f(P)$ is a homeomorphism. Finally, let $F^{\prime} \preceq f(P)$; then there is a $F \preceq P$ with $\operatorname{dim}(F)=\operatorname{dim}\left(\bar{F}^{\prime}\right)$ such that $f(F)=F^{\prime}$ (the equality follows from the proof of point (i)). Hence, $f^{-1}\left(F^{\prime}\right)=F$, which proves the lemma.

The proof of point (i) in Lemma A1 yields the following corollary.

Corollary A1. Let $P$ and $P^{\prime}$ be polytopes and $f: P \rightarrow$ $P^{\prime}$ be polyhedral. If $F$ and $F^{\prime}$ are as in the definition of a polyhedral map, then $f(F)=F^{\prime}$.

The following lemma constitutes the essential argument in the proof of Lemma A1 We remark that the proof could have been shortened by the use of simple arguments from homology theory.

Lemma A2. Let $D^{n} \subset \mathbb{R}^{n}$ be homeomorphic to a close disk and $S^{n-1} \equiv \operatorname{bd}\left(D^{n}\right)$. Let $f: D^{n} \rightarrow D^{n} \subset \mathbb{R}^{n}$ be a homeomorphism such that $f\left(S^{n-1}\right) \subseteq S^{n-1}$. Then $f\left(S^{n-1}\right)=S^{n-1}$

Proof. Following Bredon (1993, Corollary IV.19.6), we conclude that $\mathbb{R}^{n} \backslash f^{-1}\left(S^{n-1}\right)$ consists of two $(n>$ 1 ) or three $(n=1)$ components. Let $U_{1}, U_{2}$ and $U_{3}$ be these components, with $U_{3}$ possibly empty, and let $V_{i}=U_{i} \cap \operatorname{int}\left(D^{n}\right), i=1,2,3$. Then $f\left(V_{1} \cup V_{2} \cup\right.$ $\left.V_{3}\right)=\operatorname{int}\left(D^{n}\right)$, since if $z \in \operatorname{int}\left(D^{n}\right)$ then $f^{-1}(z) \in$ $\operatorname{int}\left(D^{n}\right) \backslash f^{-1}\left(S^{n-1}\right)=V_{1} \cup V_{2} \cup V_{3}$. Moreover, since $V_{1} \cup V_{2} \cup V_{3}=f^{-1}\left(\operatorname{int}\left(D^{n}\right)\right)$ we conclude by continuity that precisely one $V_{i}$, say $V_{1}$, is non-empty. Thus, $V_{1}=$ $\operatorname{int}\left(D^{n}\right) \backslash f^{-1}\left(S^{n-1}\right)$. But $\operatorname{int}\left(D^{n}\right) \cap f^{-1}\left(S^{n-1}\right)=\emptyset$, because if not, let $U \subset \operatorname{int}\left(D^{n}\right)$ be an open neighborhood of a point in the intersection, say $x$, and $\left\{x_{n}\right\} \subset U_{2}$ be a sequence converging to $x$. Then $x_{n} \in U$ for $n$ large enough, which yields the contradiction $V_{2} \neq \emptyset$. Hence, $V_{1}=\operatorname{int}\left(D^{n}\right)$, which completes the proof since $f$ is surjective.

The following three results have the appearance of being standard results from polytope theory. However, the authors have not been able to find a reference for these results, so they are included with proofs.

Lemma A3. If $H_{1}$ and $H_{2}$ are hyperplanes, then $H_{1} \cap H_{2}$ is a face of $H_{1}^{+} \cap H_{2}^{+}$.

Proof. If $H_{1} \cap H_{2}=\emptyset$, then the lemma follows. We assume that $H_{1} \cap H_{2} \neq \emptyset$ and show that there is a supporting hyperplane $C$ of $H_{1}^{+} \cap H_{2}^{+}$such that $H_{1}^{+} \cap H_{2}^{+} \subseteq C^{+}$and $H_{1} \cap H_{2}=C \cap H_{1}^{+} \cap H_{2}^{+}$. Let $H_{i} \equiv H_{i}\left(N_{i}, \alpha_{i}\right)$ for some $N_{i} \in \mathbb{R}^{n}$ and $a_{i} \in \mathbb{R}$, $i=1,2$. Suppose first that $0 \in H_{1} \cap H_{2}$, i.e., $\alpha_{1}=$ 
$\alpha_{2}=0$, and let $C \equiv\left\{x \in \mathbb{R}^{n} \mid\left\langle x, N_{1}+N_{2}\right\rangle=0\right\}$. It is clear that $H_{1}^{+} \cap H_{2}^{+} \subseteq C^{+}$and $H_{1} \cap H_{2} \subseteq C$. This shows that $H_{1} \cap H_{2} \subseteq C \cap\left(H_{1}^{+} \cap H_{2}^{+}\right)$. We show that $C \cap\left(H_{1}^{+} \cap H_{2}^{+}\right) \subseteq H_{1} \cap H_{2}$. Suppose that $x \in C \cap\left(H_{1}^{+} \cap H_{2}^{+}\right)$. Since $\left\langle x, N_{i}\right\rangle \geq 0$ for $i=1,2$ and $\left\langle N_{1}, x\right\rangle=-\left\langle N_{2}, x\right\rangle$, we have $\left\langle x, N_{i}\right\rangle=0$ for $i=1,2$. Thus, $H_{1} \cap H_{2}=C \cap\left(H_{1}^{+} \cap H_{2}^{+}\right)$. For an arbitrary $\alpha_{i}, i=1,2$, let $w \in H_{1} \cap H_{2}$ and define $G_{i} \equiv H_{i}-w$, $i=1,2$. Since $0 \in G_{1} \cap G_{2}$, there exists a hyperplane $C$ such that $G_{1}^{+} \cap G_{2}^{+} \subseteq C^{+}$and $G_{1} \cap G_{2}=C \cap\left(G_{1}^{+} \cap G_{2}^{+}\right)$. Therefore, $C_{w} \equiv C+w$ is a supporting hyperplane of $H_{1}^{+} \cap H_{2}^{+}$such that $H_{1} \cap H_{2}=C_{w} \cap\left(H_{1}^{+} \cap H_{2}^{+}\right)$. This proves the lemma.

The next lemma classifies a face of a polyhedral set, which is the intersection of two polyhedral sets, in terms of intersections of the faces of the two polyhedral sets.

Lemma A4. Let $P, Q$ be polyhedral sets. Then $G$ is a face of the polyhedral set $P \cap Q$ if and only if there are $F \preceq P$ and $L \preceq Q$ such that $G=F \cap L$.

Proof. Suppose first that $F \preceq P$ and $L \preceq Q$. If $F \cap L=$ $\emptyset$, then $G=\emptyset$ is an improper face of $P \cap Q$. If $F=P$ and $L=Q$, then $G=P \cap Q$ is again an improper face of $P \cap Q$. Suppose $F=P, L \prec Q$ and $F \cap L \neq \emptyset$. Let $H$ be a supporting hyperplane of $Q$ with $Q \subseteq H^{+}$and $H \cap Q=L$. We have $H \cap Q \cap P=L \cap P=G$ and $P \cap Q \subseteq H^{+}$. Thus, $G$ is a face of $P \cap Q$.

If $F \prec P$ and $L \prec Q$, then there are supporting hyperplanes $W$ of $P$ and $H$ of $Q$ such that $F \cap L \subseteq W \cap H$ and $P \cap Q \subseteq W^{+} \cap H^{+}$. By Lemma A3, there is a hyperplane $C$ such that $C \cap\left(W^{+} \cap H^{+}\right)=W \cap H$ and $W^{+} \cap H^{+} \subseteq C^{+}$. Since $P \cap Q \subseteq W^{+} \cap H^{+} \subseteq C^{+}$and the following equality holds:

$$
\begin{aligned}
C \cap P \cap Q=C \cap\left(W^{+} \cap H^{+} \cap P \cap Q\right) & \\
& =W \cap H \cap P \cap Q=F \cap L,
\end{aligned}
$$

it follows that $C$ is a supporting hyperplane for $P \cap Q$ and $C \cap P \cap Q=F \cap L$, thus $F \cap L$ is a face of $P \cap Q$.

We prove the converse. If $G=\emptyset$, then the lemma is proven by taking, e.g., $F=\emptyset, L=Q$. The lemma is also obvious if either $P$ or $Q$ is equal $\mathbb{R}^{n}$. It remains to prove if $G$ is a proper face of $P \cap Q$.

Let $P=\bigcap_{j=1}^{k} H_{j}^{+}$and $Q=\bigcap_{j=k+1}^{m} H_{j}^{+}$. Without loss of generality we assume that the family $\left\{H_{j}^{+} \mid j=\right.$ $1, \ldots m\}$ is irredundant, otherwise we remove redundant half-spaces from it. It follows that $P \cap Q=\bigcap_{j=1}^{m} H_{j}^{+}$. Suppose first that $G$ is a facet of $P \cap Q$, then $G=$ $H_{j} \cap(P \cap Q)$ for some $j \in\{1, \ldots, m\}$. Suppose $j \in\{1, \ldots, k\}$. Then $F \equiv H_{j} \cap P$ is a facet of $P$ and $G=F \cap Q$. Likewise, if $j \in\{k+1, \ldots, m\}$ then $G=F \cap P$, where $F$ is a facet of $Q$. By Grünbaum (2003, Theorem 2.6.5, p. 27), every proper face of a polyhedral set is an intersection of its facets. Hence, the face $G$ of
$P \cap Q$ is the intersection of facets $E_{i}(i=1, \ldots, l)$ of $P$ and $E_{i}^{\prime}\left(i=1, \ldots, l^{\prime}\right)$ of $Q$,

$$
\begin{aligned}
G & =\left(\bigcap_{i=1}^{l} E_{i}\right) \cap\left(\bigcap_{i=1}^{l^{\prime}} E_{i}^{\prime}\right) \\
& =\left(\bigcap_{i=1}^{l}\left(H_{i} \cap P\right) \cap Q\right) \cap\left(\bigcap_{i=1}^{l^{\prime}}\left(H_{i}^{\prime} \cap Q\right) \cap P\right) \\
& =\left(\bigcap_{i=1}^{l} H_{i} \cap P\right) \cap\left(\bigcap_{i=1}^{l^{\prime}} H_{i}^{\prime} \cap Q\right)=F \cap L,
\end{aligned}
$$

where $F \equiv \bigcap_{i=1}^{l} H_{i} \cap P$ is the intersection of facets of $P$. Thus, it is a face of $P$. In the same way, $L \equiv \bigcap_{i=1}^{l^{\prime}} H_{i}^{\prime} \cap Q$ is a face of $Q$.

The following lemma constitutes an essential argument in the proof of Theorem 11 We note that the proof makes implicit use of LemmaA1

Lemma A5. Let $K^{n}$ be as in Theorem $\square$ and $Q_{1}, Q_{2} \in$ $K^{n}$. Then $Q_{1} \cap Q_{2}$ is a face of $Q_{1}$ and $Q_{2}$.

Proof. We prove that $Q_{1} \cap Q_{2} \preceq Q_{1}$. For any $x \in$ $Q_{1} \cap Q_{2}$, there are $y_{1} \in U_{1} \equiv U \cap P_{1}, y_{2} \in U_{2} \equiv$ $U \cap P_{2}$ and $R \in \mathcal{R}^{1}$ such that $x=\psi\left(y_{1}\right)=\psi\left(y_{2}\right)$ and $R\left(y_{1}\right)=y_{2}$. Furthermore, from Definition 5 , if $y_{1} \in U_{1}$ and there is $R \in \mathcal{R}^{1}$ such that $y_{2} \equiv R\left(y_{1}\right) \in P_{2}$, then by point (iii), $y_{2} \in U_{2}$ and, by point (i), $\psi\left(y_{1}\right)=\psi\left(y_{2}\right)$. Let $\mathcal{R}_{1}^{2}=\left\{R \in \mathcal{R} \mid \exists y \in U_{1}\right.$ such that $\left.R(y) \in U_{2}\right\}$. From the above, it follows that $Q_{1} \cap Q_{2}=\bigcup_{R \in \mathcal{R}_{1}^{2}} \psi(\operatorname{dom}(R) \cap$ $U_{1}$ ). By Lemma 1 the set $\mathcal{R}_{1}^{2}$ is finite. Furthermore, by Lemma A4 $F \equiv \operatorname{dom}(R) \cap U_{1}$ is a face of $U_{1}$. Therefore, we can write $Q_{1} \cap Q_{2}=\bigcup_{i=1}^{k} \psi\left(F_{i}\right)$, where $F_{i} \prec U_{1}$.

We will show that

$$
\exists j \in \mathbf{k} \text { such that } F_{i} \subseteq F_{j} \forall i \in \mathbf{k} .
$$

Thereby, $Q_{1} \cap Q_{2}=\psi\left(F_{j}\right)$, but $\psi\left(F_{j}\right)$ is a face of $Q_{1}$, since $F_{j} \prec Q_{1}$ and $\left.\psi\right|_{U \cap P_{1}}$ is a restriction of a linear map. Moreover, by point (iii) of Definition 5 . $R\left(\operatorname{dom}(R) \cap U_{1}\right)=\operatorname{im}(R) \cap U_{2}$; hence, $R\left(F_{j}\right)$ is a face of $U_{2}$ and thus $\psi\left(F_{j}\right)$ is a face of $Q_{2}$.

We prove (A2) by contradiction, i.e., we assume that for any $j \in \mathbf{k}$, there is $i \in \mathbf{k}$ such that $F_{i} \backslash F_{j} \neq \emptyset$. In particular, for any $j \in \mathbf{k}$, the set $A_{j} \equiv \bigcup_{i \in \mathbf{k} \backslash\{j\}} F_{i} \backslash$ $F_{j}$ is non-empty, and we can pick $x_{j} \in A_{j}$. Let $\alpha_{i} \in$ ]0,1[, $i \in \mathbf{k}$ with $\sum_{i=1}^{k} \alpha_{i}=1$, and consider a point $x \equiv \sum_{i=1}^{k} \alpha_{i} x_{i} \in \operatorname{conv}\left(\bigcup_{i=1}^{k} F_{i}\right) \subseteq \psi^{-1}\left(Q_{1} \cap Q_{2}\right) \cap$ $U_{1}$. We show that $x \notin \bigcup_{i=1}^{k} F_{i}$, which contradicts with $Q_{1} \cap Q_{2}=\bigcup_{i=1}^{k} \psi\left(F_{i}\right)$.

Fix $j \in \mathbf{k}$, and let $H_{j}$ be a supporting hyperplane of $U_{1}$ with $F_{j}=H_{j} \cap U_{1}$ and $U_{1} \subseteq H_{j}^{+}$. If $y \in$ $A_{j} \subset \operatorname{int}\left(H_{j}^{+}\right)$, then for any $\alpha \in[0,1[$, and any $z \in$ 
$H_{j}^{+}, \alpha y+(1-\alpha) z \in \operatorname{int}\left(H_{j}^{+}\right)$. In particular, taking $\alpha=\alpha_{j}, y=x_{j}$ and $z=\frac{1}{1-\alpha_{j}} \sum_{i \in \mathbf{k} \backslash\{j\}} \alpha_{i} x_{i}$ shows that $x \in \operatorname{int}\left(H_{j}^{+}\right)$. Since this is true for any $j \in \mathbf{k}$, $x \in \bigcap_{j \in \mathbf{k}} \operatorname{int}\left(H_{j}^{+}\right)$, therefore $x \notin \bigcup_{j=1}^{k} F_{j}$.

Received: 10 April 2013

Revised: 11 November 2013 